\title{
Quantitation of oxylipins in fish and algae oil supplements using optimized hydrolysis procedures and ultra-high performance liquid chromatography coupled to tandem mass- spectrometry
}

\author{
Shiva Emami, Zhichao Zhang and Ameer Y. Taha*
}

Author affiliation:

Department of Food Science and Technology, College of Agriculture and Environmental Sciences, University of California, Davis, CA, USA

Running title: Oxidized fatty acids in fish and algae oil supplements

*Corresponding author:

Department of Food Science and Technology, College of Agriculture and Environmental Sciences, University of California Davis, One Shields Avenue, Davis, CA, USA 95616; Email: ataha@ucdavis.edu 


\section{Supplementary material}

Table S1. List of 76 oxylipins measured by UPLC-MS/MS and 9 deuterated internal standards, retention time, precursor ion, product ion and collision energy.

\begin{tabular}{|c|c|c|c|c|}
\hline Compound abbreviation & $\begin{array}{l}\text { Precursor } \\
\text { ion }(\mathbf{m} / \mathbf{z})\end{array}$ & $\begin{array}{l}\text { Product ion } \\
(\mathbf{m} / \mathbf{z})\end{array}$ & $\begin{array}{c}\text { Collision energy } \\
\text { (V) }\end{array}$ & $\begin{array}{c}\text { Retention Time } \\
\text { (RT, min) }\end{array}$ \\
\hline d-11-11(12)EpETrE & 330.2 & 167.2 & 7 & 23.5 \\
\hline d11-14,15-DiHETrE & 348.2 & 207.1 & 10 & 13.8 \\
\hline d4-6-keto-PGF1a & 373.3 & 167.1 & 19 & 4.7 \\
\hline d4-9HODE & 299.2 & 172.3 & 13 & 18 \\
\hline d4-LTB4 & 339.2 & 197.2 & 10 & 11.4 \\
\hline d4-PGE2 & 355.2 & 275.3 & 7 & 7 \\
\hline d4-TXB2 & 373.3 & 173.2 & 10 & 6 \\
\hline d6-20-HETE & 325.2 & 281.2 & 7 & 16.5 \\
\hline d8-5-HETE & 327.2 & 116.1 & 7 & 21.2 \\
\hline 10(11)-EpDPE & 343.2 & 153.2 & 4 & 23.6 \\
\hline 11(12)-EpETE & 317.2 & 167.2 & 4 & 20.5 \\
\hline 11(12)-EpETrE & 319.2 & 167.2 & 4 & 23.9 \\
\hline 11,12-DiHETrE & 337.2 & 167.1 & 13 & 14.8 \\
\hline 11-HETE & 319.2 & 167.2 & 7 & 19.5 \\
\hline 12(13)-EpOME & 295.3 & 195.2 & 7 & 22.5 \\
\hline 12,13-DiHOME & 313.2 & 183.2 & 16 & 12.7 \\
\hline 12-HEPE & 317.2 & 179.2 & 4 & 17.2 \\
\hline 12-HETE & 319.2 & 179.2 & 7 & 20.5 \\
\hline 12-oxo-ETE & 317.2 & 153.1 & 7 & 21.1 \\
\hline 13(14)-EpDPE & 343.2 & 193.2 & 4 & 23.4 \\
\hline 13-HODE & 295.2 & 195.2 & 13 & 18 \\
\hline 13-HOTrE & 293.2 & 195.1 & 10 & 15.6 \\
\hline 13-oxo-ODE & 293.2 & 195.1 & 13 & 19.3 \\
\hline 14(15)-EpETE & 317.2 & 207.2 & 4 & 20.2 \\
\hline 14(15)-EpETrE & 319.2 & 219.3 & 4 & 22.8 \\
\hline 14,15-DiHETE & 335.3 & 207.2 & 7 & 12.3 \\
\hline 14,15-DiHETrE & 337.2 & 207.1 & 10 & 13.9 \\
\hline 15(S)-HETrE & 321.2 & 221.2 & 7 & 21.1 \\
\hline 15-deoxy-PGJ2 & 315.2 & 271.2 & 4 & 16.1 \\
\hline 15-HEPE & 317.2 & 219.2 & 4 & 16.6 \\
\hline 15-HETE & 319.2 & 219.2 & 4 & 19.1 \\
\hline 15-oxo-ETE & 317.2 & 113.1 & 10 & 20.1 \\
\hline 16(17)-EpDPE & 343.2 & 233.2 & 4 & 23.3 \\
\hline 17(18)-EpETE & 317.2 & 215.2 & 4 & 19.2 \\
\hline 17,18-DiHETE & 335.3 & 247.2 & 7 & 11.7 \\
\hline 17-HDoHE & 343.2 & 281.2 & 4 & 19.5 \\
\hline 19(20)-EpDPE & 343.2 & 241.2 & 7 & 22.5 \\
\hline 20-COOH-LTB4 & 365.2 & 347.2 & 7 & 4 \\
\hline 20-HETE & 319.2 & 275.1 & 10 & 16.5 \\
\hline 20-OH-LTB4 & 351.2 & 195.2 & 13 & 5 \\
\hline 5(6)-EpETrE & 319.2 & 191.1 & 4 & 24.2 \\
\hline 5,15-DiHETE & 335.2 & 173.2 & 7 & 10.83 \\
\hline 5,6-DiHETE & 335.2 & 115.2 & 4 & 10.85 \\
\hline 5,6-DiHETrE & 337.2 & 145.1 & 7 & 16.7 \\
\hline 5-HEPE & 317.2 & 115.1 & 4 & 17.8 \\
\hline
\end{tabular}




\begin{tabular}{|c|c|c|c|c|}
\hline 5-HETE & 319.2 & 115.1 & 10 & 21.4 \\
\hline 5-охо-ЕTE & 317.2 & 273.2 & 7 & 23.4 \\
\hline 6-keto-PGF1 $\alpha$ & 369.3 & 163.2 & 22 & 4.7 \\
\hline 6-trans-LTB4 & 335.2 & 195.1 & 7 & 10.8 \\
\hline 7(8)-EpDPE & 343.2 & 113.1 & 4 & 23.8 \\
\hline 8(9)-EpETE & 317.2 & 127.2 & 4 & 20.8 \\
\hline $8(9)-\mathrm{EpETrE}$ & 319.2 & 167.2 & 4 & 23.7 \\
\hline 8,15-DiHETE & 335.2 & 235.2 & 7 & 11 \\
\hline 8,9-DiHETrE & 337.2 & 127.1 & 13 & 15.6 \\
\hline 8-HEPE & 317.2 & 155.2 & 7 & 17 \\
\hline 8-HETE & 319.2 & 155.2 & 7 & 20.4 \\
\hline 9(10)-EpOME & 295.3 & 171.1 & 7 & 22.8 \\
\hline 9,10,13-TriHOME & 329.2 & 171.1 & 16 & 6.7 \\
\hline 9,10-DiHOME & 313.2 & 201.2 & 16 & 12.42 \\
\hline 9,12,13-TriHOME & 329.2 & 211.1 & 16 & 6.6 \\
\hline 9-HETE & 319.2 & 167.2 & 7 & 19.8 \\
\hline 9-HODE & 295.2 & 171.1 & 10 & 18 \\
\hline 9-HOTrE & 293.2 & 171.2 & 4 & 15.3 \\
\hline 9-oxo-ODE & 293.2 & 185.1 & 13 & 20 \\
\hline LTB3 & 337.2 & 195.2 & 7 & 14.2 \\
\hline LTB4 & 335.2 & 195.1 & 7 & 11.4 \\
\hline LTC4 & 624.3 & 272.1 & 22 & 9.6 \\
\hline LTD4 & 495.3 & 177.1 & 13 & 7.2 \\
\hline LTE4 & 438.2 & 333.3 & 13 & 9.7 \\
\hline LXA4 & 351.2 & 115.2 & 10 & 8.1 \\
\hline PGB2 & 333.3 & 175.1 & 13 & 10 \\
\hline PGD1 & 353.3 & 317.2 & 7 & 7.2 \\
\hline PGD2 & 351.2 & 271.3 & 10 & 7 \\
\hline PGD3 & 349.3 & 269.2 & 7 & 6 \\
\hline PGE1 & 353.3 & 317.2 & 7 & 7.3 \\
\hline PGE2 & 351.2 & 271.3 & 10 & 7 \\
\hline PGE3 & 349.3 & 269.2 & 7 & 6 \\
\hline PGF $2 \alpha$ & 353.2 & 309.2 & 10 & 6.7 \\
\hline PGJ2 & 333.3 & 189.2 & 10 & 9.9 \\
\hline Resolvin E1 & 349.3 & 195 & 10 & 4.6 \\
\hline TXB2 & 369.2 & 169.1 & 10 & 6 \\
\hline 16(17)-DiHDPA & 361.5 & 233.1 & 8 & 14.5 \\
\hline 19(20)-DiHDPA & 361.5 & 273.1 & 8 & 13.8 \\
\hline 8,9-DiHETE & 335.5 & 126.9 & 12 & 12.8 \\
\hline 11,12-DiHETE & 335.5 & 167 & 10 & 12.4 \\
\hline
\end{tabular}


Table S2. Fatty acid methyl ester standard retention time

\begin{tabular}{|c|c|c|}
\hline $\mathrm{CODE}$ & Name & $\mathrm{RT}(\mathrm{min})$ \\
\hline C8:0 & Caprylic acid/Octanoic acid & 4.96 \\
\hline C10:0 & Capric Acid/Decanoic Acid & 7.296 \\
\hline C11:0 & Undecylic acid/Undecanoic acid & 8.473 \\
\hline C12:0 & Lauric Acid/Dodecanoic Acid & 9.572 \\
\hline $\mathrm{C} 13: 0$ & Tridecylic acid/Tridecanoic acid & 10.632 \\
\hline C14:0 & Myristic Acid/Tetradecanoic Acid & 11.661 \\
\hline C14:1 & Myristoleic acid & 12.005 \\
\hline C15:0 & Pentadecylic acid/Pentadecanoic Acid & 12.639 \\
\hline $\mathrm{C} 16: 0$ & Palmitic Acid/Hexadecanoic Acid & 13.636 \\
\hline C16:1 & Palmitoleic Acid & 13.914 \\
\hline C17:0 & Margaric acid/Heptadecanoic acid & 14.713 \\
\hline C18:0 & Stearic Acid/Octadecanoic Acid & 15.915 \\
\hline C18:1 trans & Elaidic Acid & 16.126 \\
\hline C18:1 cis & Oleic Acid & 16.134 \\
\hline C18:2n-6 & Linoleic Acid & 16.662 \\
\hline C18:3 n-6 & Gamma-linolenic acid & 17.066 \\
\hline C18:3n-3 & Linolenic Acid & 17.469 \\
\hline $\mathrm{C} 20: 0$ & Arachidic acid/Eicosanoic acid & 18.384 \\
\hline C20:1 n-9 & Gondoic Acid/11-eicosanoic acid & 18.601 \\
\hline $\mathrm{C} 20: 2 \mathrm{n}-6$ & 11,14-Eicosadienoic acid & 19.238 \\
\hline $\mathrm{C} 20: 3$ & Homo-gamma-linolenic acid/8,11,14-eicosatrienoic acid & 19.565 \\
\hline $\mathrm{C} 20: 4 \mathrm{n}-6$ & Arachidonic acid & 19.881 \\
\hline $\mathrm{C} 20: 3 \mathrm{n}-3$ & 11,14,17-eicosatrienoic acid & 20.057 \\
\hline $\mathrm{C} 20: 5 \mathrm{n}-3$ & Eicosapentaenoic acid (EPA) & 20.798 \\
\hline $\mathrm{C} 22: 0$ & Behenic acid/Docosanoic acid & 20.97 \\
\hline $\mathrm{C} 22: 1$ & Erucic acid & 21.251 \\
\hline $\mathrm{C} 22: 2$ & 13,16-Docosadienoic acid & 21.857 \\
\hline C22:5n-6 & Docosapentaenoic acid (DPA) & 22.941 \\
\hline $\mathrm{C} 22: 5 \mathrm{n}-3$ & Docosapentaenoic acid (DPA) & 23.431 \\
\hline $\mathrm{C} 22: 6$ & Docosahexaenoic Acid (DHA) & 23.842 \\
\hline $\mathrm{C} 24: 1$ & Nervonic acid & 23.821 \\
\hline
\end{tabular}

Table S3. Solvent gradient and flow rate in LC

\begin{tabular}{|l|l|l|l|}
\hline & Time & Solvent gradient & Flow rate \\
\hline 1 & $3.00 \mathrm{~min}$ & Solvent composition A: $60.00 \%$ B:40.00\% & $0.25 \mathrm{~mL} / \mathrm{min}$ \\
\hline 2 & $4.00 \mathrm{~min}$ & Solvent composition A: $52.00 \%$ B:48.00\% & $0.25 \mathrm{~mL} / \mathrm{min}$ \\
\hline 3 & $10.00 \mathrm{~min}$ & Solvent composition A: $40.00 \%$ B:60.00\% & $0.25 \mathrm{~mL} / \mathrm{min}$ \\
\hline 4 & $20.00 \mathrm{~min}$ & Solvent composition A: $30.00 \%$ B:70.00\% & $0.25 \mathrm{~mL} / \mathrm{min}$ \\
\hline 5 & $24.00 \mathrm{~min}$ & Solvent composition A: $15.00 \%$ B:85.00\% & $0.25 \mathrm{~mL} / \mathrm{min}$ \\
\hline 6 & $24.50 \mathrm{~min}$ & Solvent composition A: $15.00 \%$ B:85.00\% & $0.25 \mathrm{~mL} / \mathrm{min}$ \\
\hline 7 & $24.60 \mathrm{~min}$ & Solvent composition A: $0.00 \%$ B:100.00\% & $0.35 \mathrm{~mL} / \mathrm{min}$ \\
\hline 8 & $26.00 \mathrm{~min}$ & Solvent composition A: $0.00 \%$ B: $100.00 \%$ & $0.35 \mathrm{~mL} / \mathrm{min}$ \\
\hline 9 & $26.10 \mathrm{~min}$ & Solvent composition A: $65.00 \%$ B:35.00\% & $0.35 \mathrm{~mL} / \mathrm{min}$ \\
\hline 10 & $27.00 \mathrm{~min}$ & Solvent composition A: $65.00 \%$ B:35.00\% & $0.35 \mathrm{~mL} / \mathrm{min}$ \\
\hline 11 & $27.30 \mathrm{~min}$ & Solvent composition A: $65.00 \%$ B:35.00\% & $0.3 \mathrm{~mL} / \mathrm{min}$ \\
\hline
\end{tabular}


Table S4. Matrix effects (ion suppression / enhancement) of 9 deuterated surrogate standards and 72 oxylipins extracted from 2, 10, 20 or $30 \mu \mathrm{L}$ of cod liver oil in Experiment 1 ( $\mathrm{n}=1$ per oil volume).

\begin{tabular}{|c|c|c|c|c|c|}
\hline \multirow{10}{*}{$\begin{array}{l}\text { Representative } \\
\text { surrogate }\end{array}$} & & $2 \mu \mathrm{L}$ & $10 \mu \mathrm{L}$ & $20 \mu \mathrm{L}$ & $30 \mu \mathrm{L}$ \\
\hline & d-11-11(12)EpEtrE & $84 \%$ & $84 \%$ & $75 \%$ & $85 \%$ \\
\hline & d11-14,15-DiHETrE & $117 \%$ & $113 \%$ & $127 \%$ & $115 \%$ \\
\hline & d4-6-keto-PGF1a & $98 \%$ & $101 \%$ & $107 \%$ & $101 \%$ \\
\hline & d4-9HODE & $73 \%$ & $95 \%$ & $92 \%$ & $94 \%$ \\
\hline & d4-LTB4 & $105 \%$ & $96 \%$ & $108 \%$ & $110 \%$ \\
\hline & d4-PGE2 & $130 \%$ & $141 \%$ & $84 \%$ & $139 \%$ \\
\hline & d4-TXB2 & $103 \%$ & $97 \%$ & $103 \%$ & $98 \%$ \\
\hline & d6-20-HETE & $109 \%$ & $107 \%$ & $111 \%$ & $115 \%$ \\
\hline & d8-5-HETE & $110 \%$ & $106 \%$ & $107 \%$ & $110 \%$ \\
\hline \multirow{15}{*}{$\begin{array}{c}\text { d-11-11(12)- } \\
\text { EpETrE }\end{array}$} & 10(11)-EpDPE & $78 \%$ & $78 \%$ & $71 \%$ & $82 \%$ \\
\hline & 11(12)-EpETrE & $57 \%$ & $63 \%$ & $38 \%$ & $74 \%$ \\
\hline & 12(13)-ЕpOME & $81 \%$ & $83 \%$ & $51 \%$ & $85 \%$ \\
\hline & 13(14)-EpDPE & $77 \%$ & $82 \%$ & $73 \%$ & $85 \%$ \\
\hline & 14(15)-EpETE & $79 \%$ & $92 \%$ & $104 \%$ & $90 \%$ \\
\hline & 14(15)-EpETrE & $77 \%$ & $75 \%$ & $58 \%$ & $92 \%$ \\
\hline & 16(17)-EpDPE & $84 \%$ & $88 \%$ & $94 \%$ & $102 \%$ \\
\hline & 17(18)-EpETE & $63 \%$ & $92 \%$ & $107 \%$ & $95 \%$ \\
\hline & 5(6)-EpETrE & $45 \%$ & $49 \%$ & $26 \%$ & $53 \%$ \\
\hline & 7(8)-EpDPE & $63 \%$ & $68 \%$ & $55 \%$ & $78 \%$ \\
\hline & 8(9)-EpETE & $107 \%$ & $106 \%$ & $76 \%$ & $122 \%$ \\
\hline & 8(9)-EpETrE & $45 \%$ & $51 \%$ & $22 \%$ & $53 \%$ \\
\hline & 9(10)-ЕрОМЕ & $54 \%$ & $55 \%$ & $31 \%$ & $62 \%$ \\
\hline & 11(12)-EpETE & $95 \%$ & $98 \%$ & $99 \%$ & $103 \%$ \\
\hline & 19(20)-EpDPE & $95 \%$ & $93 \%$ & $109 \%$ & $99 \%$ \\
\hline \multirow{11}{*}{$\begin{array}{l}\text { d11-14,15- } \\
\text { DiHETrE }\end{array}$} & 11,12-DiHETrE & $145 \%$ & $165 \%$ & $106 \%$ & $237 \%$ \\
\hline & 12,13-DiHOME & $129 \%$ & $149 \%$ & $94 \%$ & $198 \%$ \\
\hline & 14,15-DiHETE & $137 \%$ & $129 \%$ & $127 \%$ & $125 \%$ \\
\hline & 14,15-DiHETrE & $161 \%$ & $194 \%$ & $132 \%$ & $286 \%$ \\
\hline & 17,18-DiHETE & $120 \%$ & $115 \%$ & $117 \%$ & $114 \%$ \\
\hline & 5,15-DiHETE & $116 \%$ & $125 \%$ & $74 \%$ & $139 \%$ \\
\hline & 5,6-DiHETE & $117 \%$ & $129 \%$ & $90 \%$ & $182 \%$ \\
\hline & 5,6-DiHETrE & $122 \%$ & $128 \%$ & $88 \%$ & $152 \%$ \\
\hline & 8,15-DiHETE & $90 \%$ & $91 \%$ & $49 \%$ & $107 \%$ \\
\hline & 8,9-DiHETrE & $115 \%$ & $134 \%$ & $81 \%$ & $184 \%$ \\
\hline & 9,10-DiHOME & $105 \%$ & $117 \%$ & $74 \%$ & $146 \%$ \\
\hline d4-6-keto-PGF1a & 6-keto-PGF1a & $86 \%$ & $94 \%$ & $53 \%$ & $116 \%$ \\
\hline \multirow{5}{*}{ d4-9HODE } & 13-HOTrE & $112 \%$ & $125 \%$ & $77 \%$ & $124 \%$ \\
\hline & 13-HODE & $75 \%$ & $105 \%$ & $65 \%$ & $120 \%$ \\
\hline & 17-HDoHE & $88 \%$ & $98 \%$ & $117 \%$ & $102 \%$ \\
\hline & 9-HOTrE & $96 \%$ & $109 \%$ & $69 \%$ & $134 \%$ \\
\hline & 9-HODE & $67 \%$ & $94 \%$ & $55 \%$ & $107 \%$ \\
\hline \multirow{3}{*}{ d4-LTB4 } & 20-COOH-LTB4 & $87 \%$ & $96 \%$ & $51 \%$ & $107 \%$ \\
\hline & 20-OH-LTB4 & $85 \%$ & $99 \%$ & $63 \%$ & $126 \%$ \\
\hline & 6-trans-LTB4 & $82 \%$ & $87 \%$ & $45 \%$ & $89 \%$ \\
\hline
\end{tabular}




\begin{tabular}{|c|c|c|c|c|c|}
\hline & LTB3 & $96 \%$ & $101 \%$ & $59 \%$ & $128 \%$ \\
\hline & LTB4 & $98 \%$ & $106 \%$ & $58 \%$ & $126 \%$ \\
\hline & LTC4 & $0 \%$ & $0 \%$ & $0 \%$ & $0 \%$ \\
\hline & LTD4 & $6 \%$ & $6 \%$ & $2 \%$ & $3 \%$ \\
\hline & LTE4 & $0 \%$ & $0 \%$ & $0 \%$ & $0 \%$ \\
\hline & LXA4 & $85 \%$ & $98 \%$ & $104 \%$ & $102 \%$ \\
\hline \multirow{13}{*}{ d4-PGE2 ${ }^{1}$} & 15-deoxy-PGJ2 & $87 \%$ & $89 \%$ & $54 \%$ & $135 \%$ \\
\hline & 9,10,13-TriHOME & $95 \%$ & $102 \%$ & $60 \%$ & $118 \%$ \\
\hline & 9,12,13-TriHOME & $102 \%$ & $111 \%$ & $65 \%$ & $130 \%$ \\
\hline & PGB2 & $115 \%$ & $130 \%$ & $79 \%$ & $169 \%$ \\
\hline & PGD1 & $98 \%$ & $99 \%$ & $58 \%$ & $118 \%$ \\
\hline & PGD2 & $106 \%$ & $110 \%$ & $64 \%$ & $135 \%$ \\
\hline & PGD3 & $114 \%$ & $149 \%$ & $119 \%$ & $101 \%$ \\
\hline & PGE1 & $104 \%$ & $113 \%$ & $64 \%$ & $131 \%$ \\
\hline & PGE2 & $112 \%$ & $116 \%$ & $73 \%$ & $129 \%$ \\
\hline & PGE3 & $114 \%$ & $110 \%$ & $80 \%$ & $161 \%$ \\
\hline & PGF2a & $96 \%$ & $91 \%$ & $60 \%$ & $107 \%$ \\
\hline & PGJ2 & $61 \%$ & $69 \%$ & $39 \%$ & $85 \%$ \\
\hline & Resolvin E1 & $97 \%$ & $102 \%$ & $59 \%$ & $122 \%$ \\
\hline d4-TXB2 & TXB2 & $94 \%$ & $101 \%$ & $58 \%$ & $119 \%$ \\
\hline \multirow{3}{*}{ d6-20-HETE } & 13-oxo-ODE & $59 \%$ & $90 \%$ & $77 \%$ & $91 \%$ \\
\hline & 20-HETE & $84 \%$ & $122 \%$ & $113 \%$ & $134 \%$ \\
\hline & 9-oxo-ODE & $83 \%$ & $105 \%$ & $75 \%$ & $126 \%$ \\
\hline \multirow{14}{*}{ d8-5-HETE } & 11-HETE & $88 \%$ & $116 \%$ & $71 \%$ & $151 \%$ \\
\hline & 12-HEPE & $129 \%$ & $125 \%$ & $96 \%$ & $144 \%$ \\
\hline & 12-HETE & $110 \%$ & $125 \%$ & $72 \%$ & $155 \%$ \\
\hline & 12-oxo-ETE & $101 \%$ & $118 \%$ & $64 \%$ & $142 \%$ \\
\hline & 15-HEPE & $125 \%$ & $130 \%$ & $100 \%$ & $149 \%$ \\
\hline & 15-HETE & $96 \%$ & $129 \%$ & $76 \%$ & $174 \%$ \\
\hline & 15-охо-ETE & $98 \%$ & $118 \%$ & $79 \%$ & $134 \%$ \\
\hline & 15(S)-HETrE & $123 \%$ & $130 \%$ & $89 \%$ & $161 \%$ \\
\hline & 5-HEPE & $99 \%$ & $104 \%$ & $110 \%$ & $106 \%$ \\
\hline & 5-HETE & $104 \%$ & $114 \%$ & $78 \%$ & $137 \%$ \\
\hline & 5-oxo-ETE & $65 \%$ & $67 \%$ & $36 \%$ & $76 \%$ \\
\hline & 8-HEPE & $130 \%$ & $108 \%$ & $86 \%$ & $132 \%$ \\
\hline & 8-HETE & $100 \%$ & $111 \%$ & $65 \%$ & $134 \%$ \\
\hline & 9-HETE & $116 \%$ & $121 \%$ & $71 \%$ & $154 \%$ \\
\hline
\end{tabular}

${ }^{1}$ Metabolites tagged to the d4-PGE2 are not quantifiable in this study because of the degradation of d4-PGE2 during hydrolysis. 
Table S5. Matrix effects (ion suppression / enhancement) of 9 surrogate standards and 72 oxylipins hydrolyzed using $0.25 \mathrm{M}$ sodium carbonate or sodium hydroxide in Experiment 5 ( $\mathrm{n}=1$ per base).

\begin{tabular}{|c|c|c|c|}
\hline \multirow{10}{*}{$\begin{array}{l}\text { Representative } \\
\text { surrogate }\end{array}$} & & $\begin{array}{l}2 \mu \mathrm{L} \text { hydrolyzed with } \\
\text { sodium hydroxide }\end{array}$ & $\begin{array}{c}2 \mu \mathrm{L} \text { hydrolyzed with } \\
\text { sodium carbonate }\end{array}$ \\
\hline & d-11-11(12)EpETrE & $78 \%$ & $87 \%$ \\
\hline & d11-14,15-DiHETrE & $153 \%$ & $126 \%$ \\
\hline & d4-6-keto-PGF1a & $101 \%$ & $95 \%$ \\
\hline & d4-9HODE & $120 \%$ & $92 \%$ \\
\hline & d4-LTB4 & $105 \%$ & $88 \%$ \\
\hline & d4-PGE2 & $104 \%$ & $104 \%$ \\
\hline & $\mathrm{d} 4-\mathrm{TXB} 2$ & $88 \%$ & $88 \%$ \\
\hline & d6-20-HETE & $341 \%$ & $114 \%$ \\
\hline & d8-5-HETE & $136 \%$ & $110 \%$ \\
\hline \multirow{15}{*}{$\begin{array}{c}\text { d-11- } \\
\text { 11(12)EpEtrE }\end{array}$} & 10(11)-EpDPE & $72 \%$ & $98 \%$ \\
\hline & 11(12)-EpETrE & $94 \%$ & $92 \%$ \\
\hline & 12(13)-ЕpOME & $74 \%$ & $87 \%$ \\
\hline & 13(14)-EpDPE & $72 \%$ & $94 \%$ \\
\hline & 14(15)-EpETE & $76 \%$ & $109 \%$ \\
\hline & 14(15)-EpETrE & $80 \%$ & $94 \%$ \\
\hline & 16(17)-EpDPE & $89 \%$ & $110 \%$ \\
\hline & 17(18)-EpETE & $76 \%$ & $136 \%$ \\
\hline & 5(6)-EpETrE & $143 \%$ & $142 \%$ \\
\hline & 7(8)-EpDPE & $86 \%$ & $97 \%$ \\
\hline & 8(9)-ЕpETE & $116 \%$ & $98 \%$ \\
\hline & 8(9)-EpETrE & $104 \%$ & $93 \%$ \\
\hline & 9(10)-ЕрОМЕ & $58 \%$ & $69 \%$ \\
\hline & 11(12)-EpETE & $73 \%$ & $101 \%$ \\
\hline & 19(20)-EpDPE & $91 \%$ & $114 \%$ \\
\hline \multirow{11}{*}{$\begin{array}{l}\text { d11-14,15- } \\
\text { DiHETrE }\end{array}$} & 11,12-DiHETrE & $135 \%$ & $118 \%$ \\
\hline & 12,13-DiHOME & $94 \%$ & $94 \%$ \\
\hline & 14,15-DiHETE & $142 \%$ & $120 \%$ \\
\hline & 14,15-DiHETrE & $126 \%$ & $117 \%$ \\
\hline & 17,18-DiHETE & $109 \%$ & $108 \%$ \\
\hline & 5,15-DiHETE & $115 \%$ & $103 \%$ \\
\hline & 5,6-DiHETE & $123 \%$ & $104 \%$ \\
\hline & 5,6-DiHETrE & $393 \%$ & $120 \%$ \\
\hline & 8,15-DiHETE & $69 \%$ & $96 \%$ \\
\hline & 8,9-DiHETrE & $152 \%$ & $114 \%$ \\
\hline & 9,10-DiHOME & $84 \%$ & $94 \%$ \\
\hline d4-6-keto-PGF1a & 6-keto-PGF1a & $89 \%$ & $87 \%$ \\
\hline \multirow{5}{*}{ d4-9HODE } & 13-HOTrE & $126 \%$ & $107 \%$ \\
\hline & 13-HODE & $111 \%$ & $91 \%$ \\
\hline & 17-HDoHE & $194 \%$ & $101 \%$ \\
\hline & 9-HOTrE & $150 \%$ & $97 \%$ \\
\hline & 9-HODE & $113 \%$ & $90 \%$ \\
\hline \multirow{4}{*}{ d4-LTB4 } & 20-COOH-LTB4 & $86 \%$ & $87 \%$ \\
\hline & 20-OH-LTB4 & $92 \%$ & $93 \%$ \\
\hline & 6-trans-LTB4 & $75 \%$ & $78 \%$ \\
\hline & LTB3 & $119 \%$ & $93 \%$ \\
\hline
\end{tabular}




\begin{tabular}{|c|c|c|c|}
\hline & LTB4 & $123 \%$ & $89 \%$ \\
\hline & LTC4 & $0 \%$ & $0 \%$ \\
\hline & LTD4 & $3 \%$ & $0 \%$ \\
\hline & LTE4 & $0 \%$ & $0 \%$ \\
\hline & LXA4 & $78 \%$ & $89 \%$ \\
\hline \multirow{13}{*}{ d4-PGE2 } & 15-deoxy-PGJ2 & $258 \%$ & $85 \%$ \\
\hline & 9,10,13-TriHOME & $85 \%$ & $85 \%$ \\
\hline & 9,12,13-TriHOME & $88 \%$ & $88 \%$ \\
\hline & PGB2 & $119 \%$ & $98 \%$ \\
\hline & PGD1 & $96 \%$ & $96 \%$ \\
\hline & PGD2 & $96 \%$ & $105 \%$ \\
\hline & PGD3 & $89 \%$ & $91 \%$ \\
\hline & PGE1 & $95 \%$ & $94 \%$ \\
\hline & PGE2 & $104 \%$ & $98 \%$ \\
\hline & PGE3 & $100 \%$ & $97 \%$ \\
\hline & PGF2a & $86 \%$ & $87 \%$ \\
\hline & PGJ2 & $143 \%$ & $91 \%$ \\
\hline & Resolvin E1 & $94 \%$ & $97 \%$ \\
\hline d4-TXB2 & TXB2 & $91 \%$ & $89 \%$ \\
\hline \multirow{3}{*}{ d6-20-HETE } & 13-oxo-ODE & $83 \%$ & $79 \%$ \\
\hline & 20-HETE & $237 \%$ & $104 \%$ \\
\hline & 9-oxo-ODE & $97 \%$ & $84 \%$ \\
\hline \multirow{14}{*}{ d8-5-HETE } & 11-HETE & $120 \%$ & $97 \%$ \\
\hline & 12-HEPE & $147 \%$ & $110 \%$ \\
\hline & 12-HETE & $113 \%$ & $97 \%$ \\
\hline & 12-oxo-ETE & $125 \%$ & $85 \%$ \\
\hline & 15-HEPE & $202 \%$ & $112 \%$ \\
\hline & 15-HETE & $125 \%$ & $103 \%$ \\
\hline & 15-oxo-ETE & $94 \%$ & $93 \%$ \\
\hline & 15(S)-HETrE & $135 \%$ & $116 \%$ \\
\hline & 5-HEPE & $359 \%$ & $94 \%$ \\
\hline & 5-HETE & $114 \%$ & $97 \%$ \\
\hline & 5-oxo-ETE & $90 \%$ & $88 \%$ \\
\hline & 8-HEPE & $88 \%$ & $93 \%$ \\
\hline & 8-HETE & $106 \%$ & $94 \%$ \\
\hline & 9-HETE & $138 \%$ & $105 \%$ \\
\hline
\end{tabular}


Table S6. Mean, SD and CV of oxylipins measured (pmol/ $\mu \mathrm{L}$ oil) across five different experiments where $2 \mu \mathrm{L}$ cod liver oil was hydrolyzed with $200 \mu \mathrm{L}$ of $0.25 \mathrm{M}$ sodium carbonate in the presence of extraction solvent. Inter-experimental mean, SD and CV of means from each experiment is presented in the last column.

\begin{tabular}{|c|c|c|c|c|c|c|c|c|c|c|c|c|c|c|c|c|c|c|c|c|}
\hline \multirow{2}{*}{ FA precursor } & \multirow{2}{*}{ Oxylipins } & \multicolumn{3}{|c|}{ EXP 1: $(n=3)$} & \multicolumn{3}{|c|}{ EXP2: $(n=3)$} & \multicolumn{3}{|c|}{ EXP 3: $(n=3)$} & \multicolumn{3}{|c|}{$\operatorname{EXP} 4: 4(n=3)$} & \multicolumn{3}{|c|}{ EXP5: $(n=5)$} & \multicolumn{3}{|c|}{$\begin{array}{c}\text { Inter-experimental } \\
\text { variability }\end{array}$} & \\
\hline & & Mean & $\mathrm{SD}$ & $\mathrm{CV}$ & Mean & SD & $\mathrm{CV}$ & Mean & $\mathrm{SD}$ & $\mathrm{CV}$ & Mean & SD & $\mathrm{CV}$ & Mean & $\mathrm{SD}$ & $\mathrm{CV}$ & Mean & $\mathrm{SD}$ & $\mathrm{CV}$ & \\
\hline \multirow{2}{*}{ ALA } & & 9-HOTrE & 0.69 & 0.09 & $11 \%$ & 0.40 & 0.05 & $14 \%$ & 0.77 & 0.15 & $20 \%$ & 0.80 & 0.11 & $15 \%$ & 0.28 & 0.06 & $20 \%$ & 0.59 & 0.23 & $40 \%$ \\
\hline & \multicolumn{2}{|r|}{ 13-HOTrE } & 0.73 & 0.11 & $12 \%$ & 0.50 & 0.05 & $9 \%$ & 0.65 & 0.11 & $18 \%$ & 0.85 & 0.22 & $27 \%$ & 0.33 & 0.04 & $13 \%$ & 0.61 & 0.20 & $33 \%$ \\
\hline \multirow{10}{*}{ EPA } & \multicolumn{2}{|r|}{ 5-HEPE } & 50.68 & 5.26 & $8 \%$ & 45.25 & 2.94 & $6 \%$ & 62.39 & 8.43 & $14 \%$ & 44.74 & 4.21 & $11 \%$ & 41.86 & 2.88 & $7 \%$ & 48.98 & 8.14 & $17 \%$ \\
\hline & \multicolumn{2}{|r|}{ 8-HEPE } & 4.78 & 0.78 & $13 \%$ & 5.19 & 0.05 & $1 \%$ & 6.83 & 1.37 & $20 \%$ & 9.16 & 1.42 & $16 \%$ & 2.60 & 0.64 & $24 \%$ & 5.71 & 2.45 & $43 \%$ \\
\hline & \multicolumn{2}{|r|}{ 12-HEPE } & 7.61 & 0.64 & $7 \%$ & 3.74 & 0.55 & $15 \%$ & 6.58 & 1.64 & $25 \%$ & 8.09 & 1.72 & $21 \%$ & 2.50 & 0.50 & $20 \%$ & 5.70 & 2.46 & $43 \%$ \\
\hline & \multicolumn{2}{|r|}{ 15-HEPE } & 7.41 & 0.95 & $11 \%$ & 4.73 & 0.52 & $11 \%$ & 6.63 & 1.81 & $27 \%$ & 8.10 & 1.79 & $24 \%$ & 3.83 & 0.65 & $17 \%$ & 6.14 & 1.80 & $29 \%$ \\
\hline & \multicolumn{2}{|r|}{ 8(9)-ЕpETE } & 5.35 & 1.26 & $19 \%$ & 2.92 & 2.31 & $79 \%$ & 1.50 & 0.58 & $39 \%$ & 4.21 & 0.63 & $10 \%$ & 2.91 & 1.71 & $59 \%$ & 3.38 & 1.46 & $43 \%$ \\
\hline & & 11(12)-EpETE & 4.80 & 1.36 & $21 \%$ & 2.50 & 1.80 & $72 \%$ & 1.24 & 0.62 & $50 \%$ & 5.47 & 1.60 & $41 \%$ & 2.46 & 1.71 & $60 \%$ & 3.29 & 1.77 & $54 \%$ \\
\hline & & 14(15)-EpETE & 15.80 & 4.50 & $23 \%$ & 9.14 & 4.59 & $50 \%$ & 5.00 & 3.15 & $63 \%$ & 25.46 & 9.94 & $33 \%$ & 8.94 & 7.24 & $81 \%$ & 12.87 & 8.04 & $62 \%$ \\
\hline & & 17(18)-EpETE & 185.37 & 50.12 & $22 \%$ & 102.68 & 52.59 & $51 \%$ & 51.42 & 34.67 & $67 \%$ & 328.25 & 144.03 & $37 \%$ & 85.63 & 69.09 & $81 \%$ & 150.67 & 110.82 & $74 \%$ \\
\hline & & 14,15-DiHETE & 15.03 & 2.88 & $16 \%$ & 5.25 & 0.62 & $12 \%$ & 5.97 & 0.26 & $4 \%$ & 7.59 & 0.97 & $10 \%$ & 5.99 & 0.70 & $12 \%$ & 7.97 & 4.04 & $51 \%$ \\
\hline & & 17,18-DiHETE & 22.62 & 3.65 & $13 \%$ & 8.21 & 0.64 & $8 \%$ & 12.12 & 1.74 & $14 \%$ & 11.97 & 1.40 & $13 \%$ & 8.05 & 1.04 & $13 \%$ & 12.59 & 5.94 & $47 \%$ \\
\hline & & 17-HDoHE & 21.73 & 4.68 & $18 \%$ & 9.85 & 0.54 & $5 \%$ & 17.13 & 2.82 & $16 \%$ & 24.04 & 1.89 & $5 \%$ & 5.12 & 0.81 & $16 \%$ & 15.57 & 7.97 & $51 \%$ \\
\hline & & 7(8)-EpDPE & 3.02 & 0.82 & $22 \%$ & 2.08 & 1.62 & $78 \%$ & 1.17 & 0.45 & $38 \%$ & 3.39 & 0.54 & $9 \%$ & 2.06 & 1.11 & $54 \%$ & 2.34 & 0.88 & $37 \%$ \\
\hline & & 10(11)-EpDPE & 4.58 & 0.82 & $15 \%$ & 3.07 & 2.01 & $65 \%$ & 1.42 & 0.55 & $39 \%$ & 4.51 & 1.02 & $19 \%$ & 2.57 & 1.39 & $54 \%$ & 3.23 & 1.34 & $41 \%$ \\
\hline DHA & & 13(14)-EpDPE & 5.08 & 1.09 & $18 \%$ & 3.91 & 2.11 & $54 \%$ & 2.02 & 1.04 & $52 \%$ & 8.35 & 3.10 & $33 \%$ & 3.28 & 2.25 & $68 \%$ & 4.53 & 2.41 & $53 \%$ \\
\hline & & 16(17)-EpDPE & 6.25 & 1.89 & $25 \%$ & 4.51 & 2.13 & $47 \%$ & 2.17 & 1.24 & $57 \%$ & 11.30 & 4.03 & $29 \%$ & 4.51 & 3.17 & $70 \%$ & 5.75 & 3.42 & $60 \%$ \\
\hline & & 19(20)-EpDPE & 23.45 & 7.32 & $19 \%$ & 13.94 & 5.68 & $41 \%$ & 12.14 & 4.04 & $28 \%$ & 36.84 & 9.66 & $43 \%$ & 15.03 & 6.47 & $34 \%$ & 20.28 & 10.23 & $50 \%$ \\
\hline & & 9-HODE & 2.08 & 0.40 & $16 \%$ & 0.83 & 0.14 & $17 \%$ & 1.46 & 0.30 & $21 \%$ & 1.98 & 0.46 & $21 \%$ & 0.98 & 0.14 & $14 \%$ & 1.47 & 0.57 & $39 \%$ \\
\hline & & 13-HODE & 3.76 & 0.81 & $18 \%$ & 1.68 & 0.15 & $9 \%$ & 2.32 & 0.38 & $17 \%$ & 3.12 & 0.73 & $22 \%$ & 1.83 & 0.26 & $14 \%$ & 2.54 & 0.88 & $35 \%$ \\
\hline & & 9-oxo-ODE & 0.72 & 0.19 & $22 \%$ & 0.33 & 0.02 & $6 \%$ & 0.37 & 0.03 & $7 \%$ & 0.64 & 0.24 & $38 \%$ & 0.34 & 0.07 & $21 \%$ & 0.48 & 0.18 & $38 \%$ \\
\hline & & 13-oxo-ODE & 1.94 & 0.66 & $28 \%$ & 1.18 & 0.01 & $1 \%$ & ND & - & - & 2.13 & 0.35 & $16 \%$ & 0.36 & 0.03 & $9 \%$ & 1.12 & 0.94 & $84 \%$ \\
\hline LA & & 9(10)-EpOME & 0.53 & 0.07 & $11 \%$ & 0.38 & 0.26 & $69 \%$ & 0.15 & 0.05 & $36 \%$ & 0.50 & 0.14 & $29 \%$ & 0.34 & 0.15 & $44 \%$ & 0.38 & 0.15 & $39 \%$ \\
\hline & & 12(13)-EpOME & 1.76 & 0.29 & $14 \%$ & 1.25 & 0.83 & $66 \%$ & 0.52 & 0.23 & $45 \%$ & 2.18 & 0.36 & $12 \%$ & 0.97 & 0.53 & $55 \%$ & 1.33 & 0.65 & $49 \%$ \\
\hline & & 9,10-DiHOME & 0.68 & 0.13 & $16 \%$ & 0.23 & 0.01 & $4 \%$ & 0.36 & 0.02 & $7 \%$ & 0.38 & 0.08 & $19 \%$ & 0.26 & 0.03 & $11 \%$ & 0.38 & 0.18 & $46 \%$ \\
\hline & & 12,13-DiHOME & 0.27 & 0.06 & $18 \%$ & 0.12 & 0.01 & $9 \%$ & 0.16 & 0.02 & $14 \%$ & 0.24 & 0.05 & $21 \%$ & 0.14 & 0.02 & $12 \%$ & 0.19 & 0.07 & $36 \%$ \\
\hline DGLA & & 15(S)-HETrE & 0.48 & 0.12 & $21 \%$ & 0.31 & 0.05 & $16 \%$ & 0.27 & 0.05 & $17 \%$ & 0.35 & 0.07 & $19 \%$ & 0.21 & 0.04 & $19 \%$ & 0.32 & 0.10 & $31 \%$ \\
\hline & & 5-HETE & 1.86 & 0.37 & $16 \%$ & 1.46 & 0.17 & $12 \%$ & 1.69 & 0.27 & $16 \%$ & 1.58 & 0.18 & $5 \%$ & 0.79 & 0.06 & $8 \%$ & 1.48 & 0.41 & $28 \%$ \\
\hline & & 8-HETE & 0.43 & 0.13 & $24 \%$ & ND & - & - & 0.33 & 0.06 & $18 \%$ & 0.45 & 0.08 & $11 \%$ & 0.17 & 0.02 & $12 \%$ & 0.28 & 0.19 & $69 \%$ \\
\hline & & 9-HETE & 0.30 & 0.05 & $15 \%$ & 0.07 & 0.12 & $173 \%$ & 0.23 & 0.03 & $15 \%$ & 0.24 & 0.16 & $71 \%$ & 0.12 & 0.02 & $13 \%$ & 0.19 & 0.09 & $49 \%$ \\
\hline & & 11-HETE & 0.39 & 0.09 & $19 \%$ & 0.30 & 0.05 & $16 \%$ & 0.39 & 0.08 & $20 \%$ & 0.43 & 0.09 & $12 \%$ & 0.15 & 0.02 & $14 \%$ & 0.33 & 0.11 & $34 \%$ \\
\hline & & 12-HETE & 0.27 & 0.01 & $3 \%$ & 0.14 & 0.12 & $87 \%$ & 0.26 & 0.08 & $32 \%$ & 0.33 & 0.07 & $17 \%$ & 0.11 & 0.03 & $25 \%$ & 0.22 & 0.09 & $41 \%$ \\
\hline & & 15-HETE & 0.49 & 0.07 & $12 \%$ & 0.29 & 0.06 & $22 \%$ & 0.53 & 0.12 & $23 \%$ & 0.64 & 0.16 & $24 \%$ & 0.24 & 0.06 & $27 \%$ & 0.44 & 0.17 & $39 \%$ \\
\hline & & 20-HETE & 2.51 & 0.68 & $22 \%$ & 1.26 & 0.17 & $13 \%$ & 1.41 & 0.35 & $25 \%$ & 1.80 & 0.28 & $15 \%$ & 1.35 & 0.17 & $12 \%$ & 1.67 & 0.51 & $31 \%$ \\
\hline & & 15-oxo-ETE & 0.39 & 0.04 & $9 \%$ & 0.53 & 0.09 & $16 \%$ & 0.22 & 0.03 & $11 \%$ & 0.35 & 0.10 & $23 \%$ & ND & - & - & 0.30 & 0.20 & $67 \%$ \\
\hline $\mathrm{AA}$ & & 5(6)-EpETrE & 0.22 & 0.06 & $22 \%$ & 0.15 & 0.11 & $75 \%$ & 0.06 & 0.03 & $49 \%$ & 0.21 & 0.01 & $6 \%$ & 0.14 & 0.09 & $68 \%$ & 0.15 & 0.06 & $40 \%$ \\
\hline & & 8(9)-EpETrE & 0.18 & 0.06 & $27 \%$ & 0.19 & 0.10 & $54 \%$ & 0.10 & 0.04 & $41 \%$ & 0.31 & 0.02 & $8 \%$ & 0.16 & 0.08 & $49 \%$ & 0.19 & 0.07 & $40 \%$ \\
\hline & & 11(12)-EpETrE & 0.31 & 0.08 & $20 \%$ & 0.19 & 0.10 & $51 \%$ & 0.12 & 0.04 & $39 \%$ & 0.56 & 0.19 & $28 \%$ & 0.21 & 0.12 & $58 \%$ & 0.28 & 0.17 & $62 \%$ \\
\hline & & 14(15)-EpETrE & 0.72 & 0.15 & $18 \%$ & 0.64 & 0.23 & $36 \%$ & 0.24 & 0.14 & $61 \%$ & 1.48 & 0.79 & $51 \%$ & 0.43 & 0.31 & $72 \%$ & 0.70 & 0.47 & $68 \%$ \\
\hline & & 5,6-DiHETrE & 0.22 & 0.07 & $25 \%$ & 0.10 & 0.02 & $19 \%$ & 0.10 & 0.02 & $21 \%$ & 0.12 & 0.03 & $30 \%$ & 0.12 & 0.01 & $11 \%$ & 0.13 & 0.05 & $37 \%$ \\
\hline & & 8,9-DiHETrE & 0.09 & 0.02 & $16 \%$ & ND & - & - & ND & - & - & 0.02 & 0.03 & $141 \%$ & ND & - & - & 0.02 & 0.04 & $176 \%$ \\
\hline & & 11,12-DiHETrE & 0.05 & 0.01 & $16 \%$ & 0.02 & 0.002 & $10 \%$ & 0.03 & 0.004 & $13 \%$ & 0.02 & 0.02 & $71 \%$ & 0.02 & 0.003 & $14 \%$ & 0.03 & 0.01 & $47 \%$ \\
\hline & & 14,15-DiHETrE & 0.07 & 0.02 & $24 \%$ & 0.04 & 0.004 & $10 \%$ & 0.05 & 0.01 & $18 \%$ & 0.06 & 0.004 & $8 \%$ & 0.05 & 0.005 & $11 \%$ & 0.05 & 0.01 & $26 \%$ \\
\hline & & LXA4 & 1.87 & 0.51 & $22 \%$ & 1.08 & 0.04 & $4 \%$ & 0.61 & 0.05 & $8 \%$ & 1.07 & 0.16 & $16 \%$ & 1.36 & 0.18 & $13 \%$ & 1.20 & 0.46 & $38 \%$ \\
\hline
\end{tabular}

ND: Not detected. 
Table S7. Mean, SD and CV of oxylipins measured (pmol/ $\mu \mathrm{L}$ oil) across three different experiments where $10 \mu \mathrm{L}$ cod liver oil was hydrolyzed with $200 \mu \mathrm{L}$ of $0.25 \mathrm{M}$ sodium carbonate in the presence of extraction solvent. Inter-experimental mean, SD and CV of means from each experiment are presented in the last 3 columns.

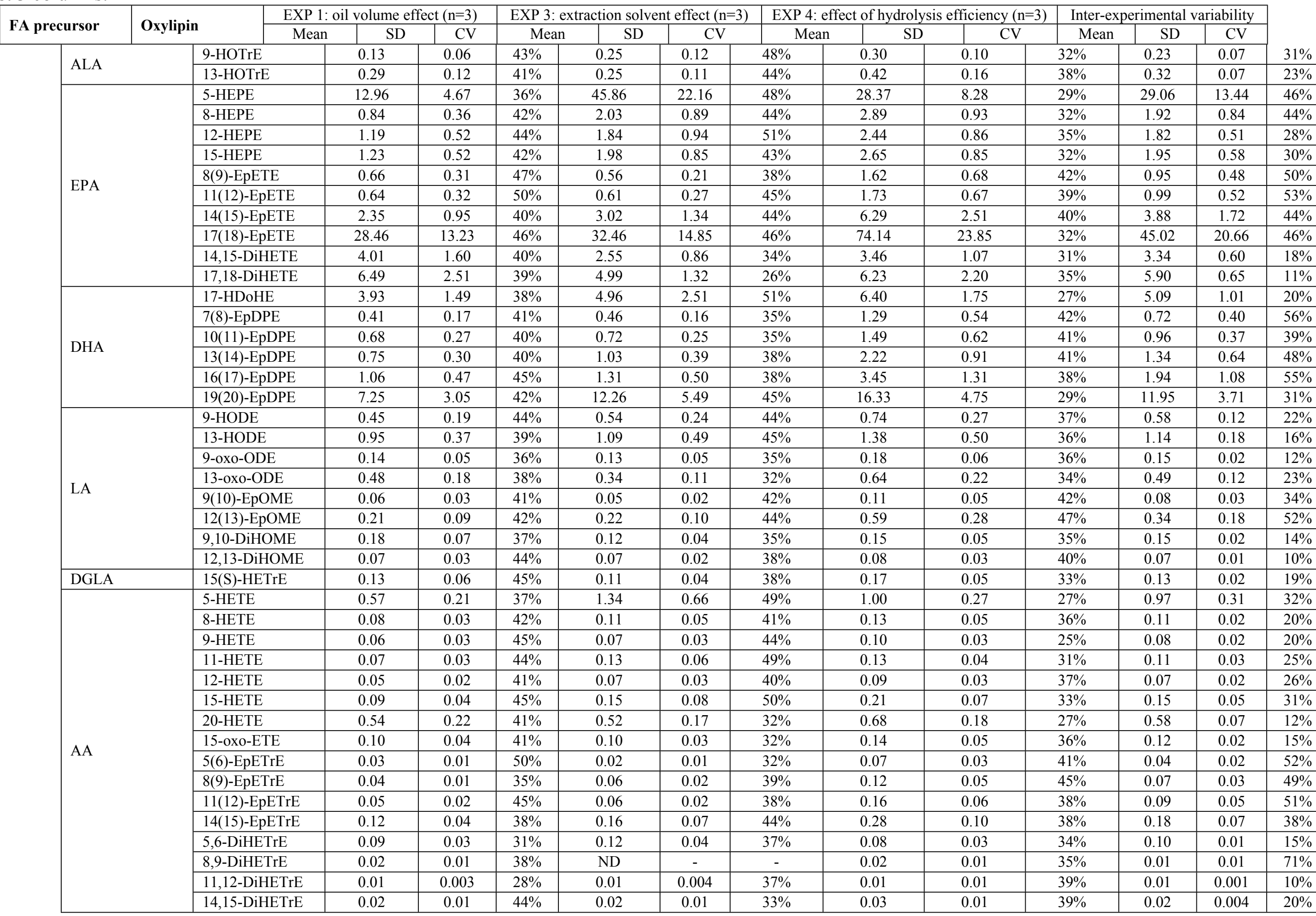


1 Table S8. Concentrations (pmol/ $\mu \mathrm{L}$ oil) of oxylipins in fish and algae oils (Experiment 6$)$. Oxylipin concentrations were determined in

$22 \mu \mathrm{L}$ of oil reconstituted in extraction solvent and hydrolyzed with $200 \mu \mathrm{L}$ of sodium hydroxide $(0.25 \mathrm{M})$. Data are presented as mean \pm

3 SD ( $n=3$ per oil) and statistically analyzed using one-way ANOVA followed by Tukey’s post-hoc test. Different letters represent

4 significant differences between the means $(p<0.05)$.

\begin{tabular}{|c|c|c|c|c|c|c|c|c|c|c|c|}
\hline \multirow{2}{*}{$\begin{array}{l}\text { FA } \\
\text { precursor }\end{array}$} & \multirow{2}{*}{$\begin{array}{l}\text { Oxylipin } \\
\text { metabolite }\end{array}$} & \multicolumn{2}{|l|}{ Fish oil 1} & \multicolumn{2}{|l|}{ Fish oil 2} & \multicolumn{2}{|l|}{ Fish oil 3} & \multicolumn{2}{|c|}{ Fish oil 4 (Cod liver oil) } & \multicolumn{2}{|l|}{ Algae oil } \\
\hline & & Mean \pm SD & $\mathrm{CV} \%$ & Mean \pm SD & $\mathrm{CV} \%$ & Mean \pm SD & $\mathrm{CV} \%$ & Mean \pm SD & $\mathrm{CV} \%$ & Mean \pm SD & $\mathrm{CV} \%$ \\
\hline ALA & 9-HOTrE & $0.97 \pm 0.26^{\mathrm{a}}$ & $27 \%$ & $0.18 \pm 0.04^{b}$ & $23 \%$ & $0.50 \pm 0.10^{b c}$ & $20 \%$ & $0.56 \pm 0.04^{\mathrm{c}}$ & $6 \%$ & $0.33 \pm 0.06^{\mathrm{bc}}$ & $18 \%$ \\
\hline ALA & 13-HOTrE & $7.41 \pm 1.34^{\mathrm{a}}$ & $18 \%$ & $3.76 \pm 0.43^{b}$ & $11 \%$ & $3.50 \pm 0.69^{b}$ & $20 \%$ & $0.40 \pm 0.04^{\mathrm{c}}$ & $11 \%$ & $0.53 \pm 0.09^{\mathrm{c}}$ & $18 \%$ \\
\hline \multicolumn{2}{|c|}{ Total ALA metabolites } & 8.38 & & 3.94 & & 4.00 & & 0.96 & & 0.86 & \\
\hline EPA & 5-HEPE & $69.93 \pm 14.01^{\mathrm{a}}$ & $20 \%$ & $8.19 \pm 2.61^{b}$ & $32 \%$ & $38.21 \pm 3.46^{\mathrm{c}}$ & $9 \%$ & $48.06 \pm 1.78^{c}$ & $4 \%$ & ND & - \\
\hline EPA & 8-HEPE & $40.86 \pm 11.13^{\mathrm{a}}$ & $27 \%$ & $2.39 \pm 0.35^{b}$ & $15 \%$ & $8.57 \pm 1.45^{b}$ & $17 \%$ & $4.63 \pm 0.23^{b}$ & $5 \%$ & $0.22 \pm 0.04^{b}$ & $18 \%$ \\
\hline EPA & 12-HEPE & $51.47 \pm 10.04^{\mathrm{a}}$ & $20 \%$ & $3.18 \pm 1.23^{b c}$ & $39 \%$ & $14.36 \pm 2.93^{\mathrm{b}}$ & $20 \%$ & $9.46 \pm 0.59^{b c}$ & $6 \%$ & $0.35 \pm 0.07^{\mathrm{c}}$ & $19 \%$ \\
\hline EPA & 15-HEPE & $100.28 \pm 20.50^{\mathrm{a}}$ & $20 \%$ & $14.95 \pm 3.05^{b}$ & $20 \%$ & $30.12 \pm 4.50^{b}$ & $15 \%$ & $16.19 \pm 0.74^{b}$ & $5 \%$ & $35.76 \pm 4.97^{b}$ & $14 \%$ \\
\hline EPA & 8(9)-EpETE & $518.00 \pm 162.93^{a}$ & $31 \%$ & $65.27 \pm 27.50^{b}$ & $42 \%$ & $68.78 \pm 25.47^{b}$ & $37 \%$ & $25.72 \pm 4.75^{b}$ & $18 \%$ & $12.00 \pm 3.21^{b}$ & $27 \%$ \\
\hline EPA & 11(12)-EpETE & $462.26 \pm 207.94^{\mathrm{a}}$ & $45 \%$ & $68.35 \pm 30.61^{b}$ & $45 \%$ & $59.36 \pm 22.83^{b}$ & $38 \%$ & $28.35 \pm 3.64^{b}$ & $13 \%$ & $1.33 \pm 0.66^{\mathrm{b}}$ & $50 \%$ \\
\hline EPA & 14(15)-EpETE & $684.39 \pm 239.29^{a}$ & $35 \%$ & $134.74 \pm 67.45^{b}$ & $50 \%$ & $118.05 \pm 38.03^{b}$ & $32 \%$ & $84.00 \pm 10.23^{b}$ & $12 \%$ & $4.04 \pm 2.07^{b}$ & $51 \%$ \\
\hline EPA & 17(18)-EpETE & $4591.86 \pm 1459.40^{\mathrm{a}}$ & $32 \%$ & $1291.92 \pm 528.06^{b}$ & $41 \%$ & $1346.99 \pm 407.48^{b}$ & $30 \%$ & $811.61 \pm 99.46^{b}$ & $12 \%$ & $49.78 \pm 29.26^{\mathrm{b}}$ & $59 \%$ \\
\hline EPA & 8,9-DiHETE & $4.80 \pm 2.09^{a}$ & $43 \%$ & $0.46 \pm 0.06^{b}$ & $13 \%$ & $0.79 \pm 0.10^{b}$ & $13 \%$ & $0.73 \pm 0.08^{b}$ & $11 \%$ & ND & - \\
\hline EPA & 8,15-DiHETE & ND & - & ND & - & $0.39 \pm 0.08$ & $21 \%$ & ND & - & ND & - \\
\hline EPA & 11,12-DiHETE & $4.54 \pm 1.79^{\mathrm{a}}$ & $39 \%$ & $0.36 \pm 0.07^{b}$ & $20 \%$ & $0.57 \pm 0.11^{b}$ & $19 \%$ & $0.48 \pm 0.06^{\mathrm{b}}$ & $13 \%$ & ND & - \\
\hline EPA & 14,15-DiHETE & $45.28 \pm 17.08^{a}$ & $38 \%$ & $4.20 \pm 1.13^{b}$ & $27 \%$ & $6.92 \pm 1.32^{b}$ & $19 \%$ & $3.21 \pm 0.29^{b}$ & $9 \%$ & ND & - \\
\hline EPA & 17,18-DiHETE & $59.24 \pm 22.71^{\mathrm{a}}$ & $38 \%$ & $6.99 \pm 0.97^{b}$ & $14 \%$ & $9.94 \pm 1.33^{b}$ & $13 \%$ & $15.12 \pm 0.72^{b}$ & $5 \%$ & $1.22 \pm 0.10^{\mathrm{b}}$ & $8 \%$ \\
\hline \multicolumn{2}{|c|}{ Total EPA metabolites } & 6632.92 & & 1600.99 & & 1703.05 & & 1047.57 & & 104.70 & \\
\hline DHA & 17-HDoHE & $100.64 \pm 44.04^{\mathrm{a}}$ & $44 \%$ & $3.55 \pm 1.12^{b}$ & $32 \%$ & $9.72 \pm 2.37^{b}$ & $24 \%$ & $6.88 \pm 0.70^{b}$ & $10 \%$ & $65.12 \pm 10.95^{\mathrm{a}}$ & $17 \%$ \\
\hline DHA & 7(8)-EpDPE & $137.36 \pm 58.14^{\mathrm{a}}$ & $42 \%$ & $22.31 \pm 7.89^{b}$ & $35 \%$ & $23.94 \pm 8.44^{b}$ & $35 \%$ & $12.80 \pm 2.35^{\mathrm{c}}$ & $18 \%$ & $104.48 \pm 41.07^{\mathrm{ab}}$ & $39 \%$ \\
\hline DHA & 10(11)-EpDPE & $138.71 \pm 54.78^{\mathrm{a}}$ & $39 \%$ & $22.96 \pm 9.25^{b}$ & $40 \%$ & $24.34 \pm 6.22^{b}$ & $26 \%$ & $15.80 \pm 2.88^{b}$ & $18 \%$ & $86.68 \pm 31.92^{\mathrm{ab}}$ & $37 \%$ \\
\hline DHA & 13(14)-EpDPE & $98.10 \pm 35.33^{\mathrm{ab}}$ & $36 \%$ & $27.89 \pm 11.95^{\mathrm{a}}$ & $43 \%$ & $30.14 \pm 7.95^{a}$ & $26 \%$ & $24.37 \pm 3.74^{\mathrm{a}}$ & $15 \%$ & $150.86 \pm 57.02^{b}$ & $38 \%$ \\
\hline DHA & 16(17)-EpDPE & $99.98 \pm 24.46^{\mathrm{ab}}$ & $24 \%$ & $33.55 \pm 12.64^{\mathrm{a}}$ & $38 \%$ & $41.42 \pm 13.37^{\mathrm{a}}$ & $32 \%$ & $32.71 \pm 3.64^{\mathrm{a}}$ & $11 \%$ & $174.75 \pm 62.69^{b}$ & $36 \%$ \\
\hline DHA & 19(20)-EpDPE & $290.80 \pm 62.92^{\mathrm{a}}$ & $22 \%$ & $79.76 \pm 36.90^{b}$ & $46 \%$ & $87.72 \pm 27.13^{b}$ & $31 \%$ & $78.87 \pm 6.65^{b}$ & $8 \%$ & $171.06 \pm 72.52^{\mathrm{ab}}$ & $42 \%$ \\
\hline DHA & 16,17-DiHDPA & $1.23 \pm 0.43^{\mathrm{a}}$ & $35 \%$ & $0.17 \pm 0.03^{c}$ & $18 \%$ & $0.46 \pm 0.09^{b c}$ & $20 \%$ & $0.40 \pm 0.04^{\mathrm{c}}$ & $10 \%$ & $1.00 \pm 0.07 \mathrm{ab}$ & $7 \%$ \\
\hline DHA & 19,20-DiHDPA & $2.45 \pm 0.73^{\mathrm{a}}$ & $30 \%$ & $0.68 \pm 0.11^{\mathrm{a}}$ & $15 \%$ & $1.45 \pm 0.24^{\mathrm{a}}$ & $17 \%$ & $1.27 \pm 0.03^{\mathrm{a}}$ & $2 \%$ & $10.88 \pm 1.36^{\mathrm{b}}$ & $13 \%$ \\
\hline \multicolumn{2}{|c|}{ Total DHA metabolites } & 869.28 & & 190.88 & & 219.20 & & 173.09 & & 764.82 & \\
\hline LA & 9-HODE & $0.82 \pm 0.38^{a}$ & $47 \%$ & $0.47 \pm 0.07^{\mathrm{a}}$ & $15 \%$ & $1.02 \pm 0.18^{\mathrm{a}}$ & $18 \%$ & $1.75 \pm 0.12^{b}$ & $7 \%$ & $2.40 \pm 0.33^{b}$ & $14 \%$ \\
\hline LA & 13-HODE & $1.78 \pm 0.12^{\mathrm{a}}$ & $6 \%$ & $0.71 \pm 0.13^{b}$ & $18 \%$ & $1.59 \pm 0.09^{\mathrm{a}}$ & $5 \%$ & $3.46 \pm 0.21^{\mathrm{c}}$ & $6 \%$ & $6.47 \pm 0.54^{\mathrm{d}}$ & $8 \%$ \\
\hline LA & 9-oxo-ODE & $0.22 \pm 0.06^{\mathrm{a}}$ & $27 \%$ & $0.15 \pm 0.02^{\mathrm{a}}$ & $14 \%$ & $0.36 \pm 0.06^{b}$ & $16 \%$ & $0.50 \pm 0.07 \mathrm{bc}$ & $13 \%$ & $0.62 \pm 0.04^{c}$ & $7 \%$ \\
\hline LA & 13-oxo-ODE & $0.23 \pm 0.13 \mathrm{ab}$ & $56 \%$ & $0.17 \pm 0.01^{b}$ & $3 \%$ & $0.26 \pm 0.10^{\mathrm{ab}}$ & $38 \%$ & $0.40 \pm 0.07^{\mathrm{a}}$ & $17 \%$ & $0.84 \pm 0.03^{c}$ & $4 \%$ \\
\hline LA & 9(10)-EpOME & $7.00 \pm 2.12^{\mathrm{ab}}$ & $30 \%$ & $2.33 \pm 1.10^{\mathrm{a}}$ & $47 \%$ & $2.66 \pm 0.88^{a}$ & $33 \%$ & $2.39 \pm 0.37^{\mathrm{a}}$ & $16 \%$ & $12.04 \pm 4.09^{b}$ & $34 \%$ \\
\hline LA & 12(13)EpOME & $9.66 \pm 1.71$ & $18 \%$ & $6.33 \pm 2.31$ & $37 \%$ & $10.40 \pm 2.97$ & $29 \%$ & $9.25 \pm 1.27$ & $14 \%$ & $5.59 \pm 1.77$ & $32 \%$ \\
\hline LA & 9,10-DiHOME & $7.44 \pm 2.99^{a b}$ & $40 \%$ & $3.85 \pm 0.95 \mathrm{bc}$ & $25 \%$ & $1.78 \pm 0.12^{\mathrm{c}}$ & $7 \%$ & $0.80 \pm 0.06^{\mathrm{c}}$ & $7 \%$ & $8.53 \pm 0.73^{a}$ & $9 \%$ \\
\hline LA & 12,13-DiHOME & $6.12 \pm 2.62^{\mathrm{a}}$ & $43 \%$ & $2.72 \pm 0.35^{b c}$ & $13 \%$ & $1.28 \pm 0.08^{\mathrm{c}}$ & $6 \%$ & $0.21 \pm 0.02^{\mathrm{c}}$ & $9 \%$ & $5.54 \pm 0.55^{\mathrm{ab}}$ & $10 \%$ \\
\hline \multicolumn{2}{|c|}{ Total LA metabolites } & 33.26 & & 16.72 & & 19.35 & & 18.75 & & 42.03 & \\
\hline DGLA & 15(S)-HETrE & $8.79 \pm 1.49^{a}$ & $17 \%$ & $1.05 \pm 0.27^{b}$ & $26 \%$ & $3.33 \pm 1.00^{c}$ & $30 \%$ & $0.41 \pm 0.02^{b}$ & $6 \%$ & $0.06 \pm 0.01^{b}$ & $22 \%$ \\
\hline AA & 5-HETE & $6.60 \pm 1.39^{a}$ & $21 \%$ & $0.52 \pm 0.08^{b}$ & $15 \%$ & $1.40 \pm 0.08^{b}$ & $6 \%$ & $0.95 \pm 0.08^{b}$ & $8 \%$ & $0.30 \pm 0.04^{b}$ & $12 \%$ \\
\hline AA & 8-HETE & $6.49 \pm 2.77^{a}$ & $43 \%$ & $0.43 \pm 0.08^{b}$ & $18 \%$ & $1.02 \pm 0.19^{b}$ & $18 \%$ & $0.50 \pm 0.07^{b}$ & $14 \%$ & ND & - \\
\hline $\mathrm{AA}$ & 9-HETE & $2.33 \pm 0.43^{\mathrm{a}}$ & $18 \%$ & ND & - & $0.60 \pm 0.16^{\mathrm{b}}$ & $27 \%$ & $0.21 \pm 0.02^{b}$ & $9 \%$ & ND & - \\
\hline AA & 11-HETE & $2.23 \pm 0.55^{\mathrm{a}}$ & $25 \%$ & $0.20 \pm 0.06^{\mathrm{bc}}$ & $29 \%$ & $0.81 \pm 0.14^{b}$ & $18 \%$ & $0.45 \pm 0.05^{\mathrm{bc}}$ & $11 \%$ & $0.06 \pm 0.01^{\mathrm{c}}$ & $21 \%$ \\
\hline AA & 12-HETE & $4.20 \pm 1.26^{\mathrm{a}}$ & $30 \%$ & $0.27 \pm 0.05^{b}$ & $20 \%$ & $0.89 \pm 0.20^{b}$ & $22 \%$ & $0.41 \pm 0.02^{b}$ & $5 \%$ & $0.10 \pm 0.01^{b}$ & $11 \%$ \\
\hline AA & 15-HETE & $4.21 \pm 0.90^{\mathrm{a}}$ & $21 \%$ & $0.18 \pm 0.07^{b}$ & $41 \%$ & $1.16 \pm 0.29^{b}$ & $25 \%$ & $0.64 \pm 0.05^{b}$ & $8 \%$ & ND & - \\
\hline AA & 20-HETE & $33.56 \pm 7.43^{\mathrm{a}}$ & $22 \%$ & $9.65 \pm 1.75^{b}$ & $18 \%$ & $9.42 \pm 2.07^{b}$ & $22 \%$ & $1.63 \pm 0.06^{b}$ & $4 \%$ & $1.34 \pm 0.23^{b}$ & $17 \%$ \\
\hline AA & 12-oxo-ETE & $0.92 \pm 0.24^{\mathrm{a}}$ & $26 \%$ & $0.09 \pm 0.03^{b}$ & $35 \%$ & $0.18 \pm 0.02^{b}$ & $9 \%$ & $0.06 \pm 0.02^{b}$ & $31 \%$ & ND & - \\
\hline
\end{tabular}




\begin{tabular}{|c|c|c|c|c|c|c|c|c|c|c|c|}
\hline $\mathrm{AA}$ & 15-oxo-ETE & ND & - & $0.10 \pm 0.05$ & $50 \%$ & $0.08 \pm 0.07$ & $87 \%$ & $0.12 \pm 0.02$ & $17 \%$ & ND & - \\
\hline $\mathrm{AA}$ & 5(6)-EpETrE & $31.27 \pm 9.03^{\mathrm{a}}$ & $29 \%$ & $1.74 \pm 0.66^{\mathrm{b}}$ & $38 \%$ & $3.50 \pm 1.08^{b}$ & $31 \%$ & $0.98 \pm 0.21^{b}$ & $21 \%$ & $0.37 \pm 0.13^{b}$ & $36 \%$ \\
\hline $\mathrm{AA}$ & 8(9)-EpETrE & $21.34 \pm 2.41^{\mathrm{a}}$ & $11 \%$ & $1.01 \pm 0.47^{b}$ & $46 \%$ & $2.82 \pm 1.09^{b}$ & $39 \%$ & $0.76 \pm 0.17^{b}$ & $22 \%$ & $0.13 \pm 0.05^{b}$ & $40 \%$ \\
\hline $\mathrm{AA}$ & 11(12)-EpETrE & $41.14 \pm 8.82^{\mathrm{a}}$ & $21 \%$ & $1.64 \pm 0.72^{b}$ & $44 \%$ & $3.90 \pm 1.22^{b}$ & $31 \%$ & $1.41 \pm 0.27^{b}$ & $19 \%$ & $0.17 \pm 0.09^{b}$ & $54 \%$ \\
\hline $\mathrm{AA}$ & 14(15)-EpETrE & $50.17 \pm 19.34^{a}$ & $39 \%$ & $4.57 \pm 3.08^{b}$ & $67 \%$ & $6.58 \pm 3.14^{b}$ & $48 \%$ & $3.40 \pm 0.41^{b}$ & $12 \%$ & $0.94 \pm 0.63^{b}$ & $67 \%$ \\
\hline $\mathrm{AA}$ & 5,6-DiHETrE & $2.24 \pm 0.56^{\mathrm{a}}$ & $25 \%$ & $0.14 \pm 0.03^{b}$ & $21 \%$ & $0.44 \pm 0.05^{b}$ & $11 \%$ & $0.27 \pm 0.02^{b}$ & $7 \%$ & $0.12 \pm 0.02^{b}$ & $16 \%$ \\
\hline $\mathrm{AA}$ & 8,9-DiHETrE & $0.44 \pm 0.22^{a}$ & $49 \%$ & ND & - & $0.09 \pm 0.02^{b}$ & $18 \%$ & ND & - & ND & - \\
\hline $\mathrm{AA}$ & 11,12-DiHETrE & $0.33 \pm 0.10^{\mathrm{a}}$ & $29 \%$ & $0.02 \pm 0.006^{b}$ & $24 \%$ & $0.06 \pm 0.01^{b}$ & $16 \%$ & $0.03 \pm 0.01^{b}$ & $18 \%$ & ND & - \\
\hline $\mathrm{AA}$ & 14,15-DiHETrE & $0.66 \pm 0.26^{\mathrm{a}}$ & $38 \%$ & $0.04 \pm 0.01^{b}$ & $14 \%$ & $0.11 \pm 0.03^{b}$ & $24 \%$ & $0.07 \pm 0.01^{b}$ & $13 \%$ & $0.03 \pm 0.01^{b}$ & $17 \%$ \\
\hline $\mathrm{AA}$ & 6-trans-LTB4 & $0.15 \pm 0.04$ & $25 \%$ & $0.12 \pm 0.10$ & $79 \%$ & $0.16 \pm 0.07$ & $41 \%$ & $0.05 \pm 0.01$ & $26 \%$ & ND & - \\
\hline $\mathrm{AA}$ & LTB4 & $0.41 \pm 0.17$ & $42 \%$ & ND & - & ND & - & ND & - & ND & - \\
\hline $\mathrm{AA}$ & LXA4 & $3.22 \pm 0.99^{a}$ & $31 \%$ & $0.91 \pm 0.17^{b}$ & $19 \%$ & $1.53 \pm 0.12^{b}$ & $8 \%$ & $0.90 \pm 0.12^{b}$ & $14 \%$ & $0.44 \pm 0.09^{b}$ & $20 \%$ \\
\hline Tota & etabolites & 211.90 & & 21.63 & & 34.77 & & 12.84 & & 3.99 & \\
\hline
\end{tabular}

$5 \quad$ ND: Not detected. No statistical analysis was performed on analytes in which there were ND metabolites in one or more experimental conditions.

6 Total oxylipins derived from each precursor fatty acid calculated as the sum of mean of individual oxylipins derived from each precursor. 
Table S9. Fatty acid concentration ( $\mathrm{mg} / \mathrm{g}$ oil) of oil supplements ( $\mathrm{n}=3)$.

\begin{tabular}{|c|c|c|c|c|c|}
\hline Fatty acid & Fish oil 1 & Fish oil 2 & Fish oil 3 & Fish oil 4 & Algae oil \\
\hline $\mathrm{C} 8: 0$ & 0 & 0 & 0 & 0 & 0 \\
\hline C10:0 & 0 & 0 & 0 & 0 & 0 \\
\hline C11:0 & 0 & 0 & 0 & 0 & 0 \\
\hline C12:0 & 0 & 0 & 0 & 0 & $5.36 \pm 0.29$ \\
\hline C13:0 & 0 & 0 & 0 & 0 & 0 \\
\hline $\mathrm{C} 14: 0$ & $0.94 \pm 0.33$ & $58.42 \pm 1.96$ & $54.62 \pm 1.12$ & $44.38 \pm 0.60$ & $234.33 \pm 13.07$ \\
\hline C14:1 & 0 & $1.91 \pm 0.10$ & $1.67 \pm 0.03$ & $2.01 \pm 0.02$ & 0 \\
\hline C15:0 & 0 & $3.68 \pm 0.09$ & $4.98 \pm 0.10$ & $3.56 \pm 0.04$ & 0 \\
\hline C16:0 & $4.72 \pm 0.13$ & $135.80 \pm 2.82$ & $132.26 \pm 2.68$ & $114.52 \pm 0.89$ & $110.79 \pm 6.23$ \\
\hline C16:1 & $2.33 \pm 0.29$ & $76.87 \pm 3.13$ & $73.55 \pm 1.51$ & $71.48 \pm 0.76$ & $1.55 \pm 0.05$ \\
\hline C18:0 & $19.94 \pm 0.42$ & $24.77 \pm 0.38$ & $27.38 \pm 0.56$ & $23.17 \pm 0.20$ & $3.80 \pm 0.22$ \\
\hline C18:1 n-9 cis & $23.69 \pm 0.41$ & $90.27 \pm 3.85$ & $87.12 \pm 1.83$ & $144.69 \pm 1.27$ & $5.68 \pm 0.87$ \\
\hline C18:1 n-7 & $12.41 \pm 0.13$ & $25.11 \pm 1.06$ & $24.60 \pm 0.53$ & $36.39 \pm 0.34$ & $1.22 \pm 0.41$ \\
\hline C18:2 n-6 & $3.65 \pm 0.04$ & $9.02 \pm 0.38$ & $11.35 \pm 0.23$ & $15.74 \pm 0.15$ & $12.04 \pm 0.69$ \\
\hline C18:3 n-6 & $3.29 \pm 0.22$ & $3.45 \pm 0.09$ & $3.46 \pm 0.05$ & $2.35 \pm 0.03$ & $1.35 \pm 0.03$ \\
\hline C18:3 n-3 & $2.25 \pm 0.14$ & $5.75 \pm 0.24$ & $6.88 \pm 0.14$ & $7.72 \pm 0.08$ & $3.41 \pm 0.20$ \\
\hline C20:0 & $8.99 \pm 0.29$ & $2.14 \pm 0.02$ & $3.89 \pm 0.08$ & $2.20 \pm 0.07$ & $0.78 \pm 0.06$ \\
\hline C20:1 n-9 & $32.48 \pm 0.39$ & $7.26 \pm 0.29$ & $10.91 \pm 0.33$ & $89.46 \pm 0.84$ & 0 \\
\hline$C 20: 2$ n-6 & $3.71 \pm 0.24$ & $1.16 \pm 0.15$ & $1.40 \pm 0.05$ & $2.46 \pm 0.03$ & 0 \\
\hline C20:3 & $2.77 \pm 0.09$ & $0.98 \pm 0.06$ & $1.07 \pm 0.17$ & $0.74 \pm 0.02$ & $0.95 \pm 0.05$ \\
\hline$C 20: 4 n-6$ & $28.37 \pm 0.44$ & $6.67 \pm 0.26$ & $12.03 \pm 0.25$ & $6.01 \pm 0.06$ & $3.55 \pm 0.20$ \\
\hline$C 20: 3 n-3$ & $1.51 \pm 0.08$ & $0.53 \pm 0.02$ & $0.79 \pm 0.03$ & $1.16 \pm 0.06$ & 0 \\
\hline$C 20: 5 n-3$ & $445.99 \pm 1.53$ & $153.65 \pm 6.08$ & $151.30 \pm 3.15$ & $100.44 \pm 1.19$ & $2.59 \pm 0.16$ \\
\hline $\mathrm{C} 22: 0$ & 0 & 0 & 0 & 0 & 0 \\
\hline $\mathrm{C} 22: 1$ & $12.73 \pm 0.58$ & $1.73 \pm 0.05$ & $8.19 \pm 0.12$ & $57.44 \pm 0.93$ & 0 \\
\hline $\mathrm{C} 22: 2$ & 0 & 0 & 0 & 0 & 0 \\
\hline$C 22: 5 n-6$ & $5.63 \pm 0.16$ & $2.19 \pm 0.07$ & $3.18 \pm 0.06$ & 0 & $74.99 \pm 4.38$ \\
\hline$C 22: 5 n-3$ & $27.94 \pm 0.30$ & $16.10 \pm 0.66$ & $15.36 \pm 0.33$ & $12.48 \pm 0.12$ & 0 \\
\hline $\mathrm{C} 22: 6$ & $172.33 \pm 1.47$ & $101.26 \pm 3.64$ & $100.24 \pm 2.14$ & $106.76 \pm 1.24$ & $398.01 \pm 22.91$ \\
\hline $\mathrm{C} 24: 1$ & 0 & 0 & 0 & 0 & 0 \\
\hline$\sum \mathrm{SFA}$ & 34.58 & 224.81 & 223.13 & 187.84 & 355.05 \\
\hline$\sum$ MUFA & 83.64 & 203.14 & 206.03 & 401.48 & 8.45 \\
\hline$\sum$ PUFA & 697.44 & 300.76 & 307.05 & 255.86 & 496.88 \\
\hline$\sum \mathrm{n}-6$ & 47.42 & 23.47 & 32.48 & 27.31 & 92.88 \\
\hline$\sum \mathrm{n}-3$ & 650.02 & 277.28 & 274.57 & 228.55 & 404.01 \\
\hline
\end{tabular}

SSFA: sum of mean of saturated fatty acids C8:0, C10:0, C11:0, C12:0, C13:0, C14:0, C15:0, C16:0, C18:0, C20:0, C22:0. ¿MUFA: sum of mean of monounsaturated fatty acids C16:1, C18:1 n-7, C18:1 n-9 cis, C20:1 n-9, C22:1; ¿PUFA: sum of mean of polyunsaturated fatty acids C18:2 n-6, C18:3 n-3, C18:3 n-6, C20:2 n-6, C20:4 n-6, C20:3 n-3, C20:5 n-3, C22:2, C22:5 n-3, C22:5 n-6, C22:6; $\mathrm{n}$-6: sum of mean of C18:2 n-6, C18:3 n-6, C20:2 n-6, C20:4 n-6, C22:2, C22:5 n-6; En-3: sum of mean of C18:3 n-3, C20:3 n-3, C20:5 n-3, C22:5 n-3, C22:6. 
Figure S1. Figure S1. Effect of adding extraction solvent (methanol with $0.1 \%$ acetic acid and $0.1 \%$ BHT) with hydrolysis reagent on oxylipin peak area (Experiment 3 ). Peak areas of oxylipins and their representative deuterated surrogates in 2 and $10 \mu \mathrm{L}$ of fish oil with or without addition of extraction solvent before hydrolysis are shown for metabolites derived from a) DHA, b) EPA, c) AA, d) LA-ALA-DGLA and e) surrogate standards.
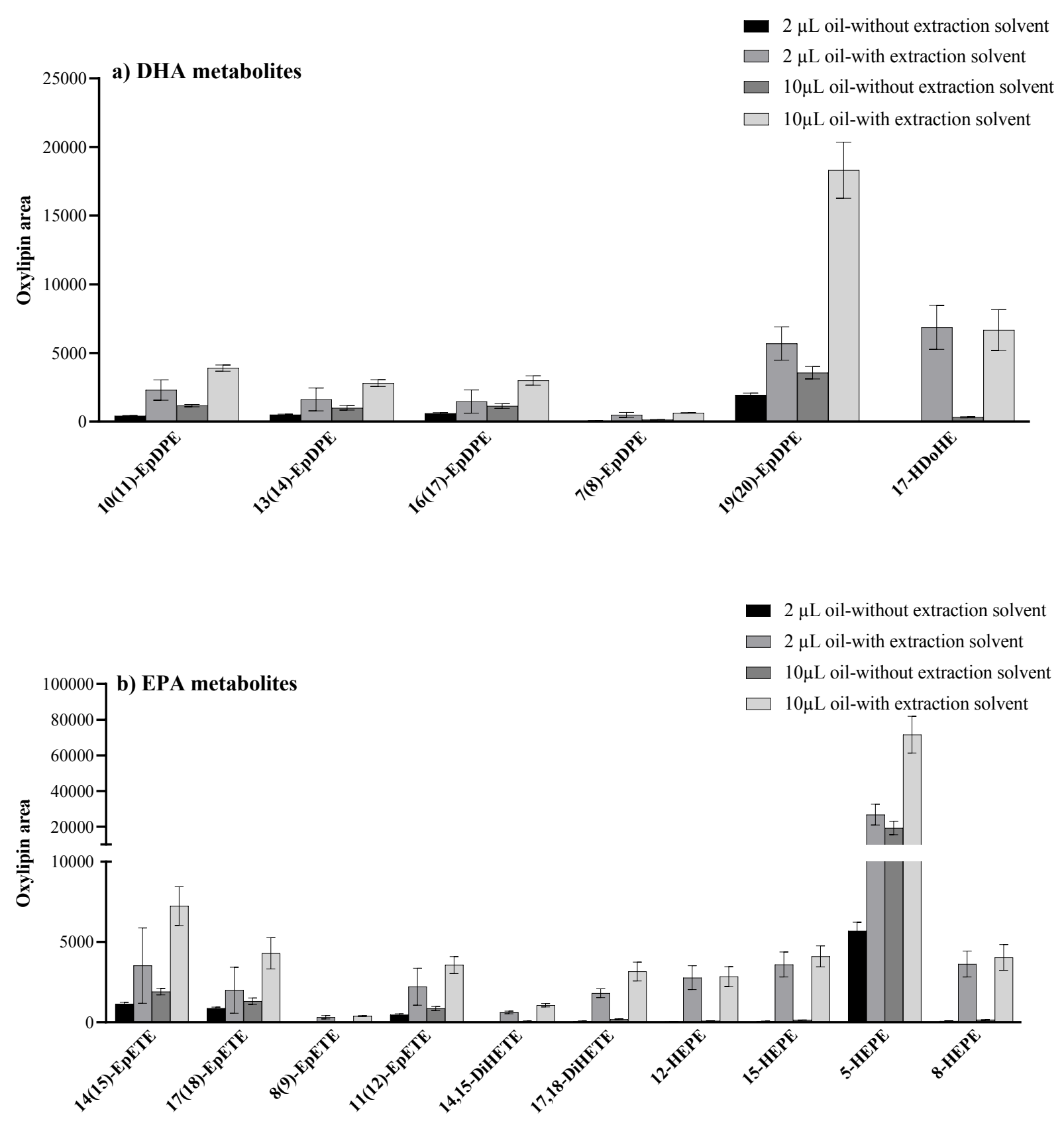
$2 \mu \mathrm{L}$ oil-without extraction solvent $2 \mu \mathrm{L}$ oil-with extraction solvent
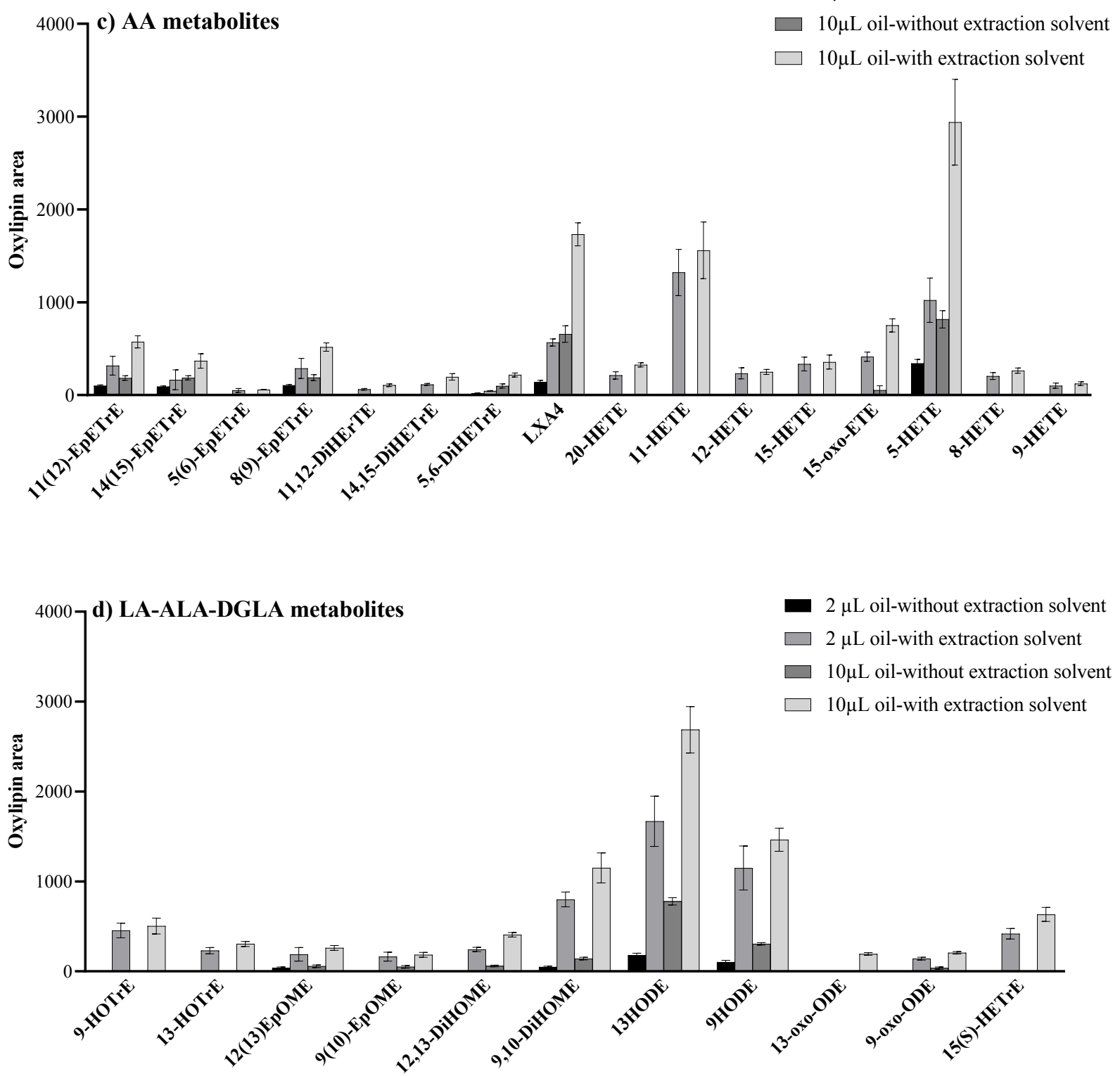


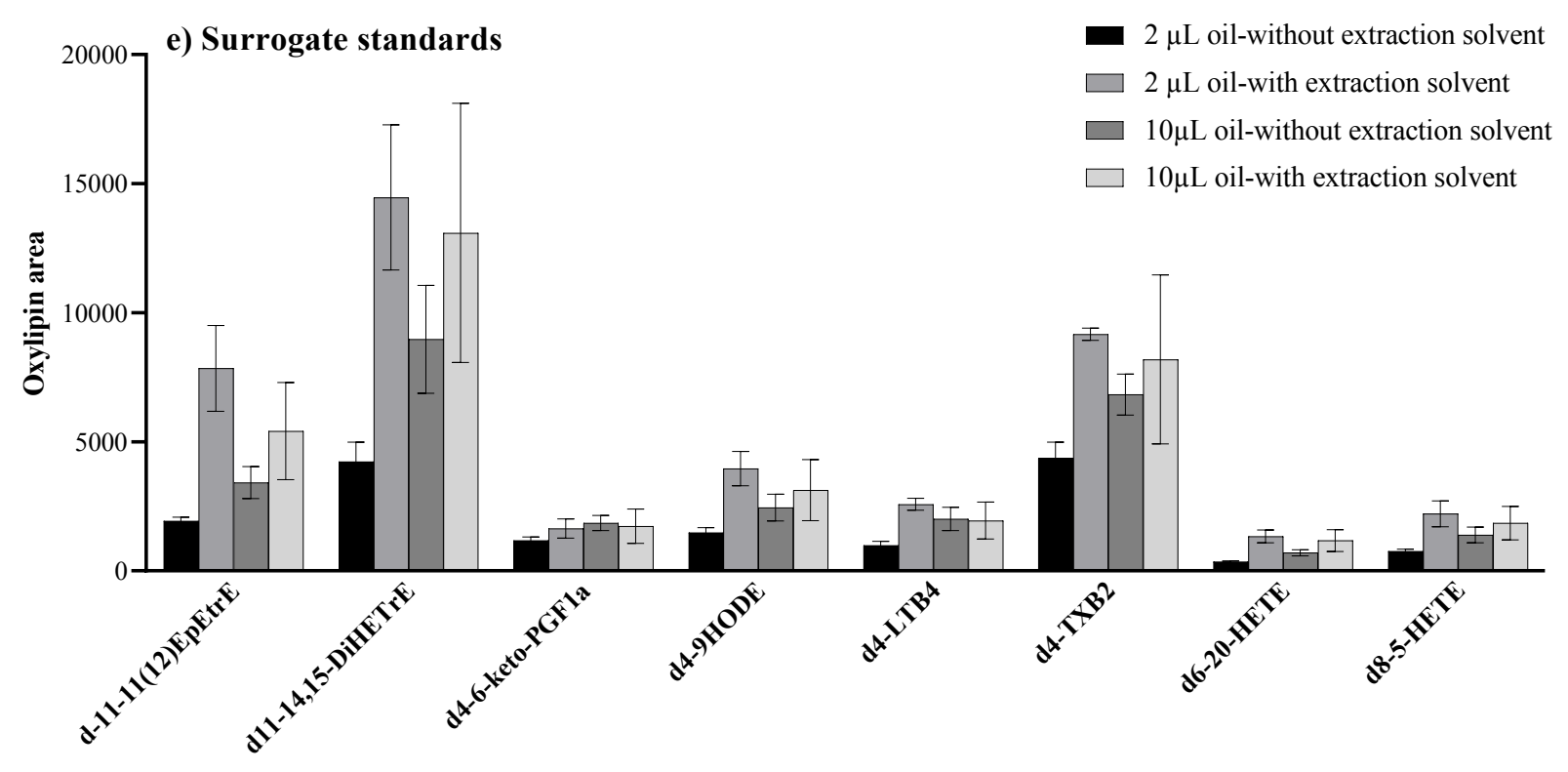


Figure S2. Representative UPLC-MS/MS chromatogram of pure oxylipin standards $(0.25-5 \mu \mathrm{M})$ dissolved in methanol.The analytes are (in order of appearance): 20-COOH-LTB4, 6-ketoPGF1a, d4-6-keto-PGF1a, Resolvin E1, 20-OH-LTB4, d4-TXB2, PGD3, PGE3, TXB2, 9,12,13TriHOME, 9,10,13-TriHOME,PGF2a, d4-PGE2, PGD2, PGE2, PGD1, PGE1, LTD4, LXA4, LTC4, LTE4, PGJ2, PGB2, 8, 15-DiHETE, 6-trans-LTB4, LTB4, 5, 15-DiHETE, 5,6-DiHETE, 17,18-DiHETE, d4-LTB4, 14,15-DiHETE, 11,12-DiHETE, 12,13-DiHOME, 8,9-DiHETE, 9,10DiHOME, d11-14,15-DiHETrE, 14,15-DiHETrE, 19,20-DiHDPA, LTB3, 16,17-DiHDPA, 11,12-DiHETrE, 9-HOTrE, 8,9- DiHETrE, 13-HOTrE, 15-deoxy-PGJ2, d6-20-HETE, 20-HETE, 15-HEPE,5,6-DiHETrE, 8-HEPE, 12-HEPE, 5-HEPE, 13-HODE, d4-9-HODE, 9-HODE, 15HETE, 17,18-EpETE, 13-oxo-ODE, 17-HDoHE, 11-HETE, 9-oxo-ODE, 15-oxo-ETE, 14,15EpETE, 8-HETE, 12-HETE, 11(12)-EpETE, 8(9)-EpETE, 9-HETE, 15(S)-HETrE, d8-5-HETE, 12-oxo-ETE, 5-HETE, 12(13)-EpOME, 19(20)EpDPE, 9(10)-EpOME, 14,15-EpETrE, 16(17)EpDPE, 5-oxo-ETE, 13(14), EpDPE, d11-11(12)-EpETrE, 10(11)-EpDPE, 11(12)-EpETrE, 8(9)-EpETrE, 7(8)-EpDPE, 5(6)- EpETrE.

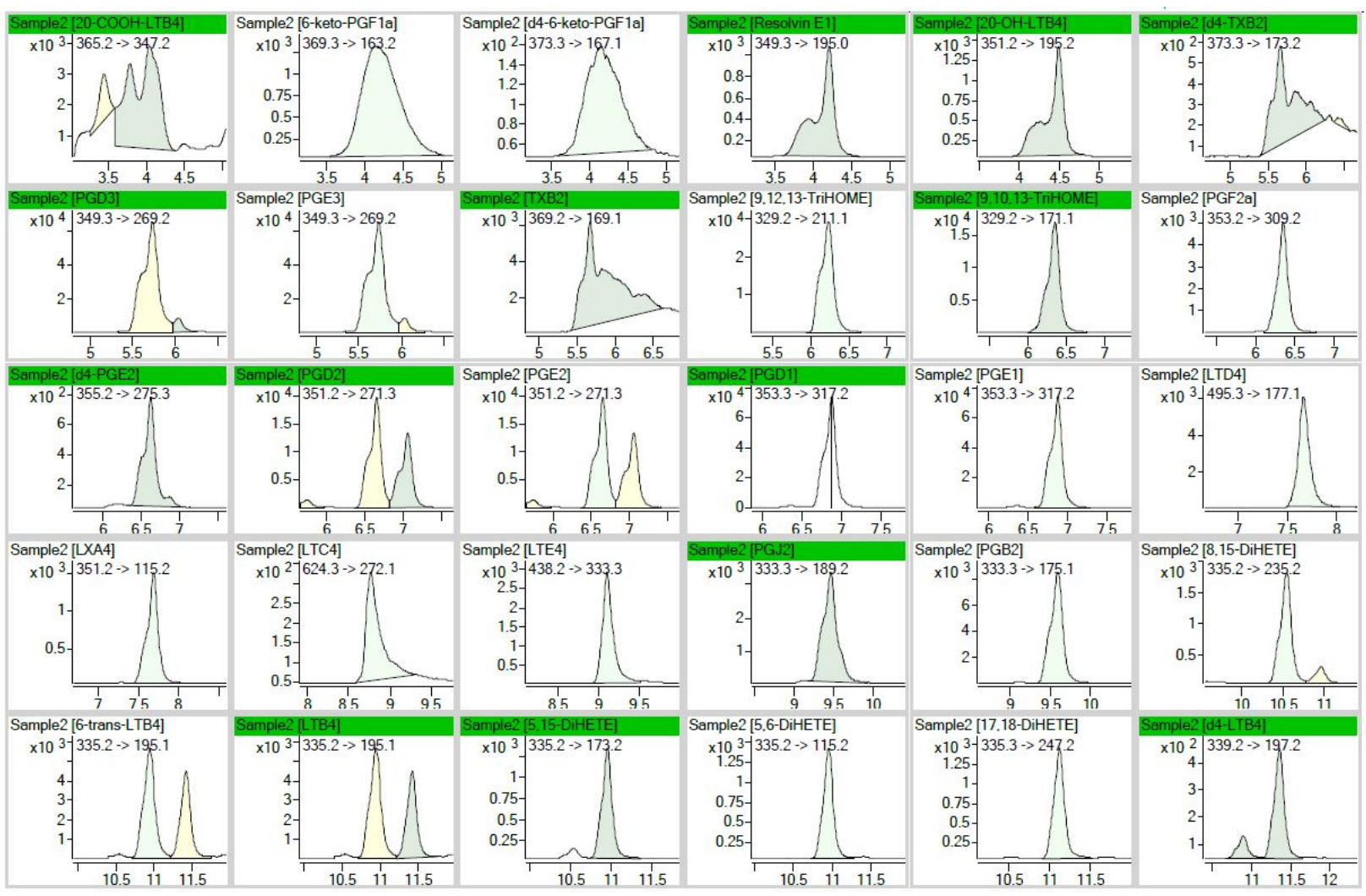




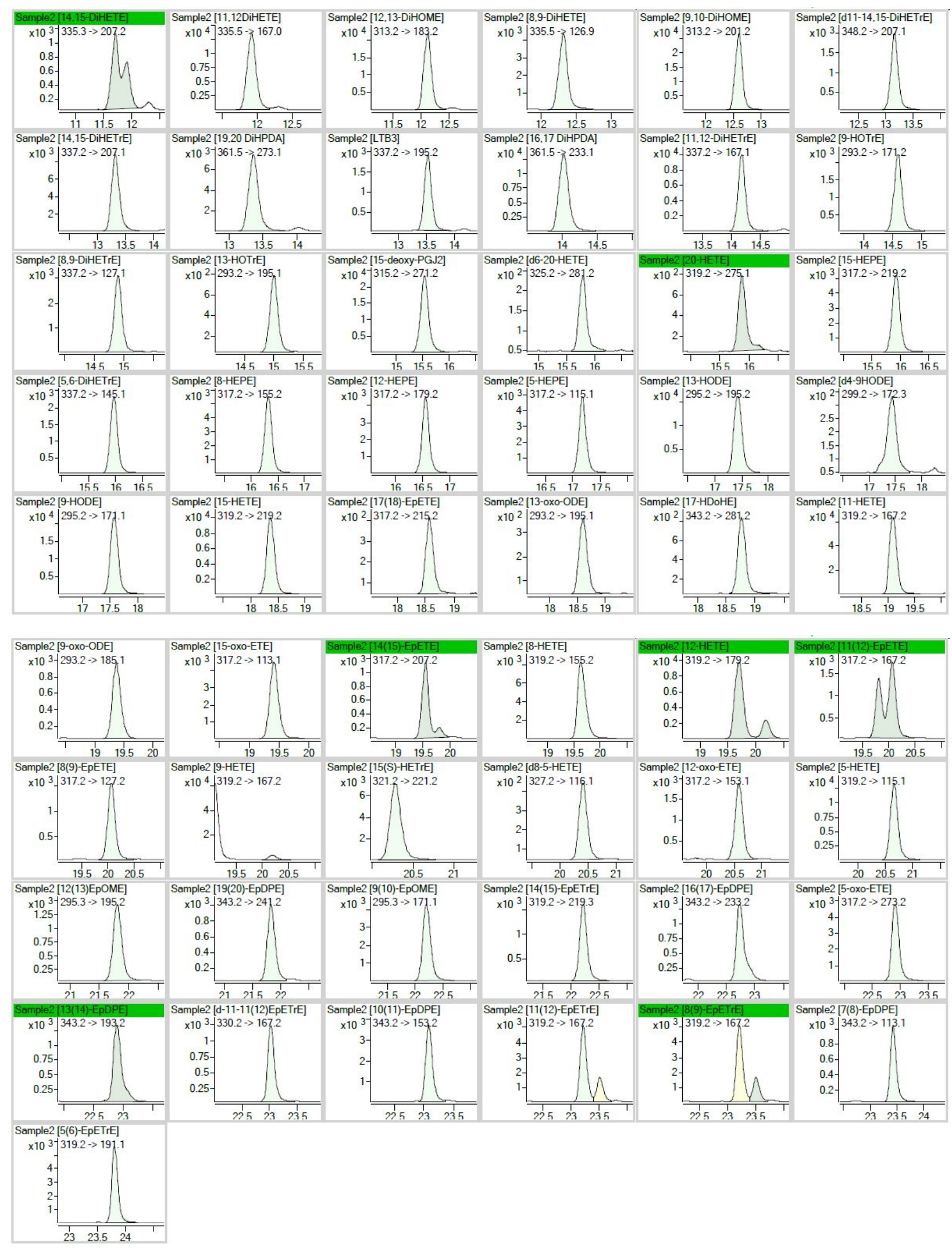


Figure S3. Representative UPLC-MS/MS chromatogram of 76 oxylipins and 9 surrogates in a fish oil sample (fish oil 3, Experiment 6). The analytes are (in order of appearance): 20-COOHLTB4, 6-keto-PGF 1a, d4-6-keto-PGF1a, Resolvin E1, 20-OH-LTB4, d4-TXB2, PGD3, PGE3, TXB2, 9,12,13-TriHOME, 9,10,13-TriHOME,PGF2a, d4-PGE2, PGD2, PGE2, PGD1, PGE1, LTD4, LXA4, LTC4, LTE4, PGJ2, PGB2, 8, 15-DiHETE, 6-trans-LTB4, LTB4, 5,15-DiHETE, 5,6-DiHETE, 17,18-DiHETE, d4-LTB4, 14,15-DiHETE, 11,12-DiHETE, 12,13-DiHOME, 8,9DiHETE, 9,10-DiHOME, d11-14,15-DiHETrE, 14,15-DiHETrE, 19,20-DiHDPA, LTB3, 16,17DiHDPA, 11,12-DiHETrE, 9-HOTrE, 8,9- DiHETrE, 13-HOTrE, 15-deoxy-PGJ2, d6-20-HETE, 20-HETE, 15-HEPE,5,6-DiHETrE, 8-HEPE, 12-HEPE, 5-HEPE, 13-HODE, d4-9-HODE, 9HODE, 15-HETE, 17,18-EpETE, 13-oxo-ODE, 17-HDoHE, 11-HETE, 9-oxo-ODE, 15-oxoETE, 14,15-EpETE, 8-HETE, 12-HETE, 11(12)-EpETE, 8(9)-EpETE, 9-HETE, 15(S)-HETrE, d8-5-HETE, 12-oxo-ETE, 5-HETE, 12(13)-EpOME, 19(20)EpDPE, 9(10)-EpOME, 14,15EpETrE, 16(17)-EpDPE, 5-oxo-ETE, 13(14), EpDPE, d11-11(12)-EpETrE, 10(11)-EpDPE, 11(12)-EpETrE, 8(9)-EpETrE, 7(8)-EpDPE, 5(6)- EpETrE.

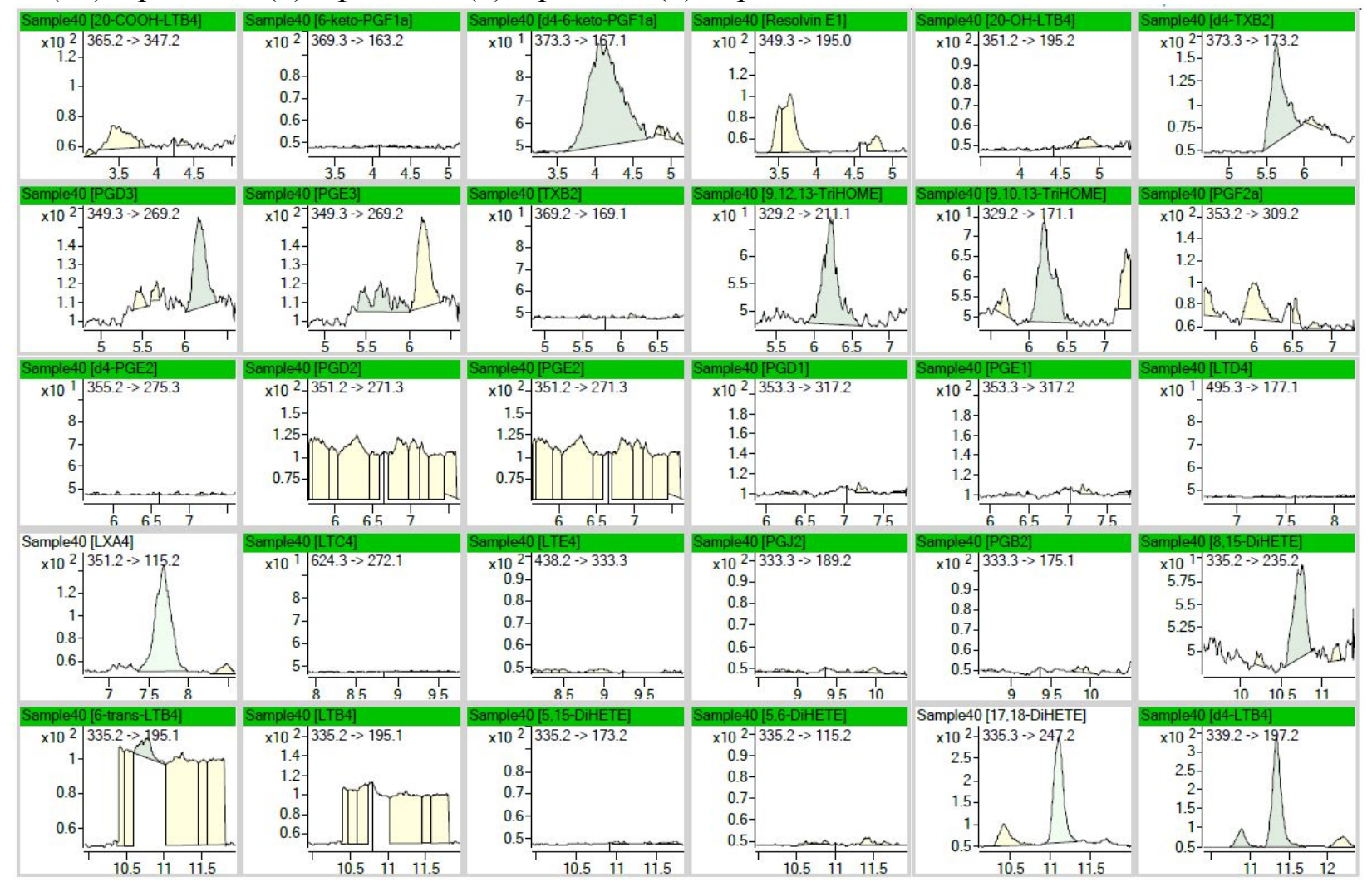




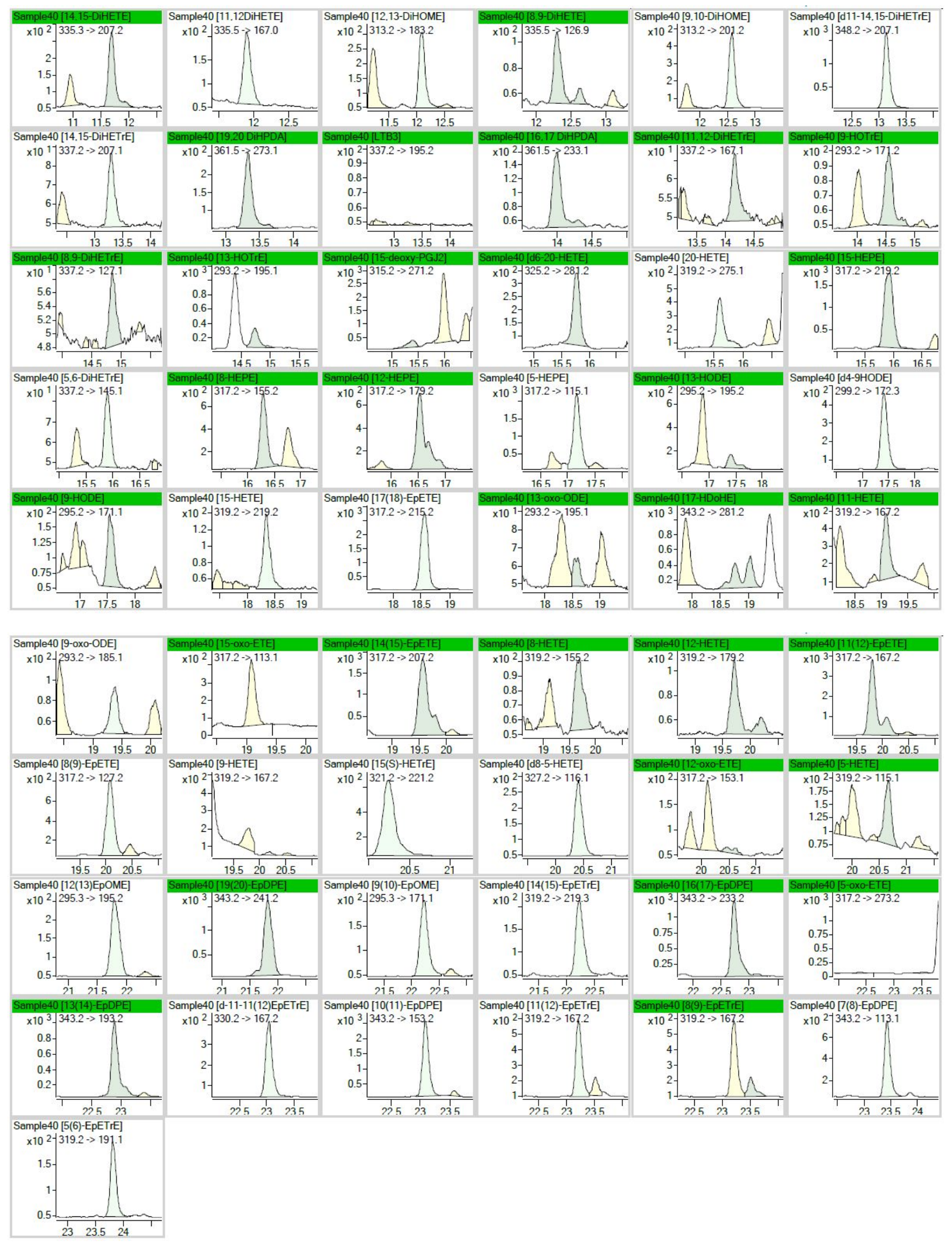


Figure S4. Representative GC chromatogram of fatty acids detected in fish oil 4 (cod liver oil).

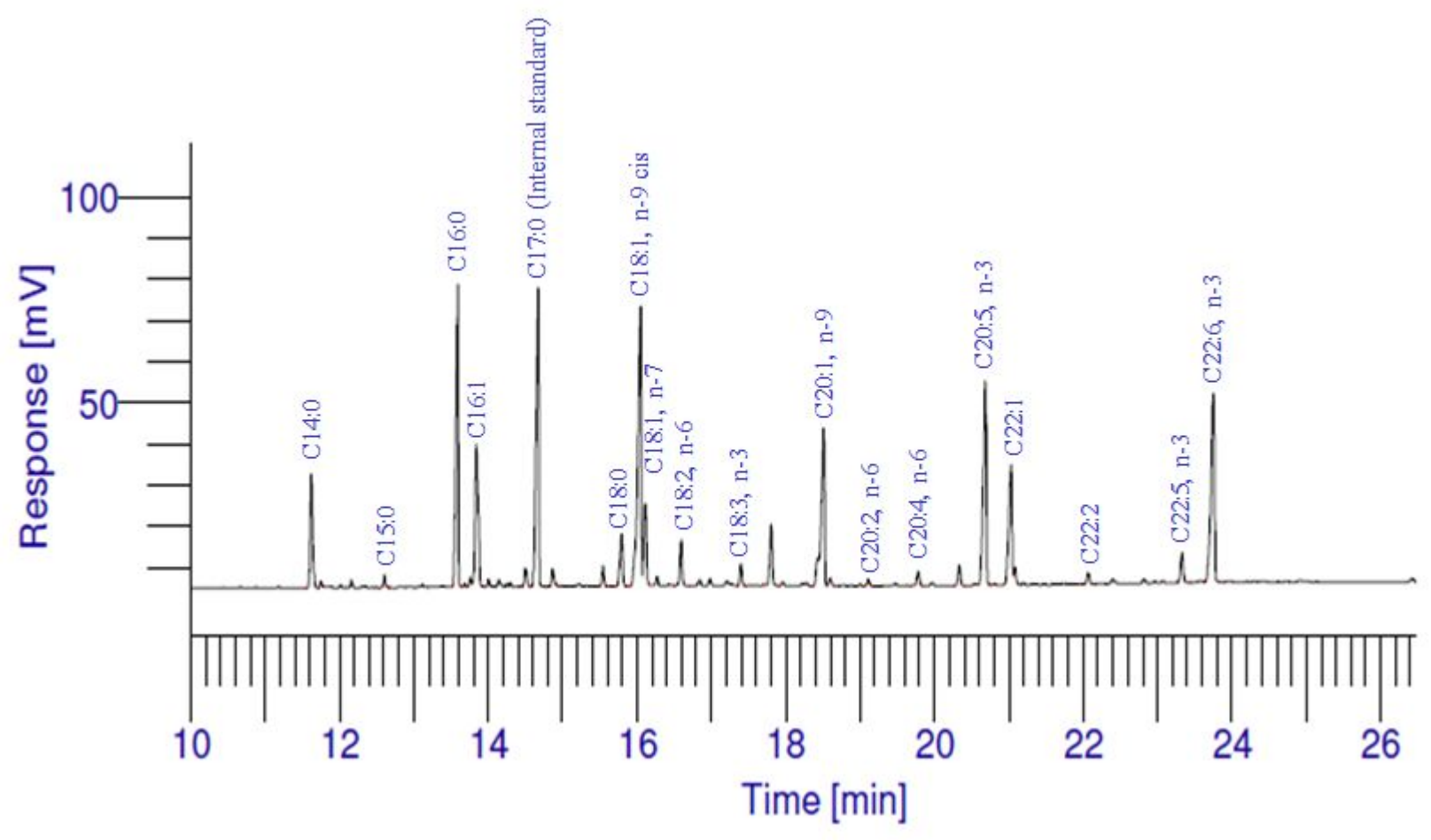

